This item was submitted to Loughborough's Research Repository by the author.

Items in Figshare are protected by copyright, with all rights reserved, unless otherwise indicated.

\title{
Switching dynamics of doped CoFeB trilayers and a comparison to the quasistatic approximation
}

\section{PLEASE CITE THE PUBLISHED VERSION}

http://dx.doi.org/10.1103/PhysRevB.87.174416

\section{PUBLISHER}

(C) American Physical Society

VERSION

VoR (Version of Record)

\section{LICENCE}

CC BY-NC-ND 4.0

\section{REPOSITORY RECORD}

Forrester, D. Michael, F.V. Kusmartsev, and Endre Kovacs. 2019. "Switching Dynamics of Doped Cofeb Trilayers and a Comparison to the Quasistatic Approximation”. figshare. https://hdl.handle.net/2134/12785. 


\title{
Switching dynamics of doped $\mathrm{CoFeB}$ trilayers and a comparison to the quasistatic approximation
}

\author{
Michael Forrester and Feodor Kusmartsev \\ Department of Physics, School of Science, Loughborough University, Leicestershire LE11 3TU, United Kingdom \\ Endre Kovács \\ Department of Physics, University of Miskolc, H-3515 Miskolc, Hungary \\ (Received 6 September 2012; revised manuscript received 22 April 2013; published 10 May 2013)
}

\begin{abstract}
The investigation of the switching times of the magnetization reversal of two interacting $\mathrm{CoFeB}$ nanomagnets, with dimensions small enough to maintain a single-domain structure, has been carried out. A quasistatic approximation is shown to give valid results and to compare well to the damped dynamical solutions of the Landau-Lifshitz-Gilbert equations. The characteristics of the switching are shown in the associated hysteresis loops and we build a complete phase diagram of the various parallel, antiparallel, and scissoring states of the magnetization in terms of the coupling energy between the nanomagnets, magnetic anisotropy, and the interaction with an applied magnetic field. The phase diagram summarizes the different kinds of hysteresis associated with the magnetization reversal phenomena. The switching fields and times are estimated and the vulnerabilities of the magnetic phases to thermally induced magnetic field variations are examined. The stability of the phases is a fine balance between intrinsic and extrinsic magnetism and we examine its precarious nature. Our work identifies the structures that have the most robust magnetization states and hence a design ethic for creating nanomagnetic heterostructures with outstanding magnetoresistance properties based upon the two magnetic elements.
\end{abstract}

DOI: 10.1103/PhysRevB.87.174416

PACS number(s): 75.75.Fk

\section{INTRODUCTION}

For many years nanomagnets have been used in the data storage industry. Magnetic random access memory (MRAM) chips store data using nanomagnets in a nonvolatile way. ${ }^{1}$ For storage applications the nanomagnets have to exhibit stable and switchable states in order for the direction of their magnetic moments to represent information as binary logic. Single-domain magnets are stable enough against thermal fluctuations in order to enable them for use as logic or memory elements. These elements constrain the development of extra domains by being small enough to do so, with the critical size before domain wall formation typically being at length scales of around $200 \mathrm{~nm}$ or less. The single-domain structure allows one to describe the atomic moments in a nanomagnet as one macrospin. In general, the nanomagnets become superparamagnetic when the geometry is made smaller than $10 \mathrm{~nm}$. Herein, we describe elongated ferromagnetic nanomagnets that are on the order of about $100 \mathrm{~nm}$ in length.

It is clear that the future use of nanomagnets in a broader scope of applications is on the horizon. In particular, the delivery of nanomagnets to cancerous cells in humans or animals in a controlled manner is the focus of an intense research effort. ${ }^{2}$ The movement in and out of cells by particles is also of crucial importance and an interdisciplinary effort is required to understand the mechanisms of transport. Designing the correct structure to attack cancer cells has to be motivated in a large part by finding the optimal geometry for movement of particles through the body. Thus, any design of nanomagnet must be optimized against this prerequisite. In light of this, it is essential for those undertaking this work to be able to understand the response of the nanomagnet to an applied magnetic field because a balance against functionality may have to be met.

In this work, we describe the dynamics of two coupled single-domain nanomagnets, which may be taken to be amorphous $\mathrm{CoFeB}$ compounds, using the Landau-LifshitzGilbert (LLG) equation. Analytical results are given and compared to the numerical solutions of the LLG equations. When the nanomagnets are in close proximity the exchange coupling [this refers in particular to RudermanKittel-Kasuya-Yosida (RKKY) interaction ${ }^{3-5}$ ] dominates the form of the coupling. At large separation $(>10 \mathrm{~nm})$ of the nanomagnets the coupling comes directly from dipole-dipole interaction.

In all applications of nanomagnetic heterostructures the dynamical behavior under an external magnetic field is very nontrivial. ${ }^{6}$ In order to thermalize the system to an energy minimum, dissipation has to be included in the study of the magnetization reversal process. Thus, one seeks to find the optimal conditions for the operation of any device, e.g., the reading and writing in MRAM or the magnetic stimulation of biofunctionalized nanomagnets to induce cancer cell death. These conditions include the optimal frequency range, the dissipation, the shape and size of the nanomagnet, and the separation between particles. Herein, a strong shape anisotropy is adopted to constrain the magnetic moments to predominantly lie in the $x-y$ plane.

Besides this, a much weaker $y$-axis shape anisotropy is also taken into account. The easy axis lies along the longest length of the nanomagnet, the $x$ direction, and an external magnetic field is applied parallel to it. A quasistatic analysis, similar to that conducted in Ref. 7, proves to be a valid approximation to the hysteresis profile associated with the dynamical evolution of the magnetic moments in the two nanomagnets. The quasistatic solutions are compared to the dynamical results that are found using the LLG equations. In the quasistatic analysis the system is always in a local energy minimum and only when this minimum changes to a saddle point does the system immediately jump into an adjacent available minimum. 
In reality, however, the jumps need some characteristic time to happen that depends on the physical properties of the system. Therefore, the system is not always in a local minimum. The speed of these processes depends on the characteristic time during which the particles change the direction of their magnetization, i.e., the time scale of the magnetization reversal. For example, in order to write information into the memory of a device, one needs to change the orientation of the magnetic moments, i.e., a magnetization reversal. This can be performed by the application of a pulsed magnetic field or by a microwave/RF field.

In this paper we present a theoretical study that compares the quasistatic and dynamical methods of analysis for a synthetic multilayer consisting of two nanomagnets. We show that the comparison is good for a range of Gilbert damping strengths and examine the effects of perturbations to the system. In the first section we describe the model and the energies associated with the nanomagnets. We then go on to give the description of the quasistatic approximation and develop the phase diagrams that elucidate the interdependence of the coupling, anisotropic, and Zeeman energy interactions of the nanomagnets, based upon its principles. The validity of the approximation is then scrutinized against dynamical results. We examine the switching times and fields associated with the dynamical evolution and the instabilities to the magnetic phases that are brought about by chaotic transient behavior and the resulting switching fluctuations.

\section{THE MAGNETIC FIELDS AND ENERGIES OF MONODOMAIN NANOMAGNETS}

The CoFeB nanomagnets are of particular interest for practical implementation as memory or logic elements due to the fact that the saturation magnetization $\left(M_{S}\right)$ associated with them can be adjusted by doping with chromium or vanadium (see, for example, Ref. 8). If the nanomagnets have high shape anisotropy then a lower $M_{s}$ is viable and the in-plane shape anisotropy energy barrier remains high enough. ${ }^{9}$ The energy barrier associated with shape anisotropy is dependent upon the square of the saturation magnetization multiplied by the demagnetization factors that are intrinsically linked with the geometry of the nanomagnet. Shape anisotropy mainly stems from surface effects and is taken as the dominant form of anisotropy in these nanomagnets. Thus, if $M_{s}^{2}$ is high and the nanomagnet has large shape anisotropy, then there is a very high energy barrier between the stable states of the nanomagnet. In the case of $\mathrm{CoFeB}$ compounds, doping can be used to reduce the saturation magnetization, with the consequence that this energy barrier decreases. To maintain the height of the energy barrier this can be compensated for by increasing the aspect ratio of the nanomagnet, i.e., making it more elongated and increasing the shape anisotropy. Neglecting any induced anisotropic effects, the shape anisotropy energy $\epsilon_{s h}$ is given by

$$
\epsilon_{s h}=-\mu_{0} V\left(N_{x} M_{x i}^{2}+N_{y} M_{y i}^{2}+N_{z} M_{z i}^{2}\right) .
$$

In the above equation, the demagnetization factors associated with the $x, y$, and $z$ axes are given by $N_{x}, N_{y}$, and $N_{z}$, respectively. Each nanomagnet in the system is assumed to have the same demagnetization factors. The magnetization vector is defined as $\mathbf{M}_{i}=M_{s} \mathbf{m}_{i}=M_{s}\left(m_{x i}, m_{y i}, m_{z i}\right)=$
$M_{s}\left(\cos \varphi_{i} \sin \theta_{i}, \sin \varphi_{i} \sin \theta_{i}, \cos \theta_{i}\right)$ in the above and throughout the text (the index $i$ denotes the first or second nanomagnet, $i=$ "1" or "2"). Typically, the magnetic saturation fields of magnetic multilayers are smaller than that of an individual layer of the magnetic material on account of the spacer material and magnetic interfacial exchange coupling. ${ }^{10}$ Frequently ruthenium is used as the interlayer between the magnetic layers in $\mathrm{CoFeB}$ (e.g., Ref. 10).

Each nanomagnet has volume $V=(\pi / 4) l_{x} l_{y} l_{z}$, where $l_{x}$, $l_{y}$, and $l_{z}$ are the lengths along the $x, y$, and $z$ axes, respectively. The cross section in the $x-y$ plane is elliptical and each nanomagnet is an elliptical cylinder. The height $l_{z}$ is $2 \mathrm{~nm}$ throughout this work. For practical applications, such as spin transfer torque MRAM, multilayers of magnetic elements combat thermal instabilities that can affect the magnetic information in single-layer devices. This increased stability is due to the increase in magnetic volume and the reduced switching current density. ${ }^{11}$

Ellipsoidal nanomagnets that are relatively elongated in the $x$ direction, compared to the other axes, have $x$-component demagnetization factors tending to zero. For example, for a very long cylindrical rod that lies parallel to $x, N_{y}=N_{z}=0.5$ and $N_{x}=0$ (when the cross section through the $y-z$ plane is circular). In this study we examine the magnetic properties of elongated nanoscale structures that have their longest axis along the $x$ direction. The energy equation for the system of two nanomagnets is

$$
E=-\mu_{0} V\left[J \mathbf{M}_{1} \cdot \mathbf{M}_{2}+\mathbf{H} \cdot\left(\mathbf{M}_{1}+\mathbf{M}_{2}\right)\right]+\epsilon_{s h},
$$

where the $J$ is a dimensionless coupling parameter. A negative or positive value of $J$ indicates antiferromagnetic or ferromagnetic coupling, respectively. The two ferromagnetic layers are separated from each other by a normal metal spacer layer in a synthetic antiferromagnetic (SAF) or synthetic ferromagnetic (SF) arrangement. ${ }^{12}$ The thickness of this nonmagnetic interlayer dictates whether the coupling of the nanomagnets is ferromagnetic or antiferromagnetic. This is in accordance with the RKKY interaction theory. The RKKY mechanism becomes dominant when the exchange coupling between localized moments and conduction electrons is sufficiently strong. ${ }^{13}$ On average the spins of the conduction electrons are unpolarized, but close to each localized moment the spins must obtain a preferred orientation (which fluctuates with distance from the moment). Interaction between the arising spin-density oscillation and another localized moment will lead to the second moment coupling to the first either ferromagnetically or antiferromagnetically depending on distance. In SAF structures the thickness of the interlayer is manipulated to enforce an antiferromagnetic coupling. Huang et al. ${ }^{14}$ demonstrated the manipulation of the coupling energy in amorphous CoFeB$\mathrm{Ru}-\mathrm{CoFeB}$ SAFs with different $\mathrm{Ru}$ thicknesses and found that the spacer layer of thickness $1.2 \mathrm{~nm} \leqslant t_{\mathrm{Ru}} \leqslant 1.5 \mathrm{~nm}$ resulted in antiferromagnetic coupling. Increasing the thickness from $t_{\mathrm{Ru}}=1.5 \mathrm{~nm}$ to $t_{\mathrm{Ru}}=1.8 \mathrm{~nm}$ saw the emergence of ferromagnetic coupling. The antiferromagnetic coupling energy is $J_{e x}=-0.05$ to $-0.01 \mathrm{erg} / \mathrm{cm}^{2}$ (Refs. 14 and 15) in SAF structures.

The applied magnetic field to these structures is denoted by $\mathbf{H}=M_{s} \mathbf{h}=M_{s}\left\{h_{x}, h_{y}, h_{z}\right\}$. Also, for convenience we write $\{e, a, b\}=2\left\{N_{x}, N_{y}, N_{z}\right\}$. Now, in order to derive the equations 
of motion of the magnetic moments we use the LLG equation ${ }^{16}$

$$
\frac{\partial \mathbf{M}_{i}}{\gamma \partial t}=\left[\mathbf{M}_{i} \times \mathbf{H}_{\mathrm{eff}, i}\right]-\frac{\alpha}{M_{s}}\left[\mathbf{M}_{i} \times\left[\mathbf{M}_{i} \times \mathbf{H}_{\mathrm{eff}, i}\right]\right],
$$

where $\gamma$ is the gyromagnetic ratio. The last term of the equation introduces Gilbert damping into the model. The Gilbert damping parameter $\alpha$ is usually around $0.005-0.03$ for doped $\mathrm{CoFeB} .{ }^{17}$ Here, the effective field is given by

$$
\mathbf{H}_{\mathrm{eff}, i}=-\frac{1}{\mu_{0} V} \frac{\partial E}{\partial \mathbf{M}_{i}} .
$$

Upon expansion of Eq. (3), we divide the resulting form by $M_{s}^{2}$ and define time as $t=\tau / \gamma M_{s}$ to get a dimensionless form of the LLG equation. We divide all the dimensionless parameters $\left(J, e, a, b\right.$, and $\left.h_{x, y, z}\right)$ by $N_{x}$, so that

$$
\widetilde{J} \rightarrow \frac{J}{N_{x}}, \quad \widetilde{e} \rightarrow 2, \quad \widetilde{a} \rightarrow \frac{a}{N_{x}}, \quad \tilde{b} \rightarrow \frac{b}{N_{x}}, \quad \widetilde{\mathbf{h}} \rightarrow \frac{\mathbf{h}}{N_{x}}
$$

For brevity, in the rest of the text we will drop the tilde above each parameter with the understanding that whenever a numerical value for them is stated it has been normalized by $N_{x}$. To investigate the magnetization characteristics we solve (3) to obtain $\partial \varphi_{i} / \partial \tau$ and $\partial \theta_{i} / \partial \tau$. We then solve the resulting four coupled first-order partial differential equations using an adaptive Runge-Kutta-Cash-Karp algorithm. In the dynamical analysis featured in this work, we apply an oscillating magnetic field in the direction of the longest axis of the nanomagnets (the $x$ direction). The SAF stack proves to be susceptible to a broad range of frequencies and for our illustrative purposes we chose to analyze the magnetic response in a $1 \mathrm{GHz}$ field, unless otherwise stated. The profile of this field changes as

$$
h_{x}(\tau)=h=h_{a} \cos f \tau,
$$

where $f=f_{\text {applied }} / \gamma M_{s}$ is the frequency in dimensionless units and $h_{a}$ is the amplitude of the field. The use of radiowave frequencies is highly desirable for controlling nanomagnets in biological tissue, for example. Radio frequency energy can safely penetrate tissue throughout the body due its low tissue-specific absorption rates. ${ }^{18}$

\section{THE STATIC APPROXIMATION FOR MONODOMAIN NANOMAGNETS}

The use of quasistatic methods to understand nanomagnets is a common strategy. As the height of the nanomagnet is typically less than $10 \mathrm{~nm}$ the magnetization dynamics generally occur close to the plane of the film. Out-of-plane oscillations do, however, take place, particularly approaching applied magnetic field strengths close to a change in state of the magnetization. ${ }^{19}$ The following quasistatic method allows one to obtain solutions for magnetization dynamics in the vicinity of stable and metastable states in the energy landscape by making the approximation that the magnetic moments are confined to move in the plane that lies perpendicular to the direction of the height. Thus, $\theta_{1,2}=\pi / 2$ and the energy equation reduces to

$$
\begin{aligned}
E_{r}= & \frac{E}{\mu_{0} M_{s} V}=-J \cos \left(\varphi_{1}-\varphi_{2}\right) \\
& -\frac{a}{2}\left(\sin ^{2} \varphi_{1}+\sin ^{2} \varphi_{2}\right)-h\left(\cos \varphi_{1}+\cos \varphi_{2}\right) .
\end{aligned}
$$

Putting $\varphi_{1}=-\varphi_{2}$ and $\varphi_{2}=\varphi$, i.e., an antiparallel alignment of the magnetizations of two nanomagnets,

$$
\frac{\partial E_{r}}{\partial \varphi}=(2 J-a) \cos \varphi+h=0 .
$$

This leads to

$$
\varphi=\arccos \left(\frac{h}{a-2 J}\right) .
$$

If we had stipulated that $\varphi_{1}=\varphi_{2}$, then the above equation would be the same other than that coupling term $J$ would have vanished. The Hessian matrix is now used to define the stability and character of the extrema in the energy landscape of the two coupled nanomagnets. The entries in the Hessian matrix are of the form $\partial^{2} E_{r} / \partial \varphi_{i, j}^{2}$, where the indices $i$ and $j$ relate to nanomagnets 1 and 2, respectively, and hence the position in the matrix. After solving for the extremal points, one then inserts the solutions into the Hessian matrix. If the resulting value is positive, then the found solution is associated with stable local minima or unstable local maxima. To determine what are the energy minima of the system, the distinction of the absolute maxima and minima is made by additionally finding values of $\partial^{2} E_{r} / \partial \varphi_{1,1}^{2}>0$. This kind of analysis is known as the second derivative test for functions of two variables. If the determinant of the Hessian matrix is zero, then the test is inconclusive. This means that the stability criterion is determined by the zeros of the determinant of the Hessian matrix. Taking the determinant of the Hessian matrix and setting it to zero, we find the critical points of stability that relate to the extrema of the system. Thus, it is found that for $\varphi_{1}=-\varphi_{2}$,

$$
h_{\rightarrow \rightarrow \rightleftharpoons \nearrow \searrow}=-(2 J-a)
$$

and

$$
h_{\leftarrow \leftarrow \rightleftharpoons \swarrow}=(2 J-a) .
$$

These equations give the critical lines of stability for transitions between parallel and scissored states. The arrows in the indices of $h$ are used to denote the directions of the magnetizations in each nanomagnet (e.g., $\rightarrow \rightarrow$ are for the parallel magnetizations along the longest axes of the nanomagnets) and the $\rightleftharpoons$ denote the critical transition. Throughout we use the word "state" for a specific characteristic configuration of the nanomagnetic moments. Because of the existence of more subtle structures in the same type of configurations (such as antiparallel or parallel or scissored) we use another term, "phase," to identify these differences. We found that for each phase there is a specific shape of the magnetization hysteresis. Then the number of different phases we have is equal to the number of different types of magnetic hysteresis displayed in the system. The critical lines of stability can be seen in Fig. 1 as the lines that separate the regions of intermediate hue (brown online) that are representative of parallel states and those that are black (scissored states). Similarly, the critical 

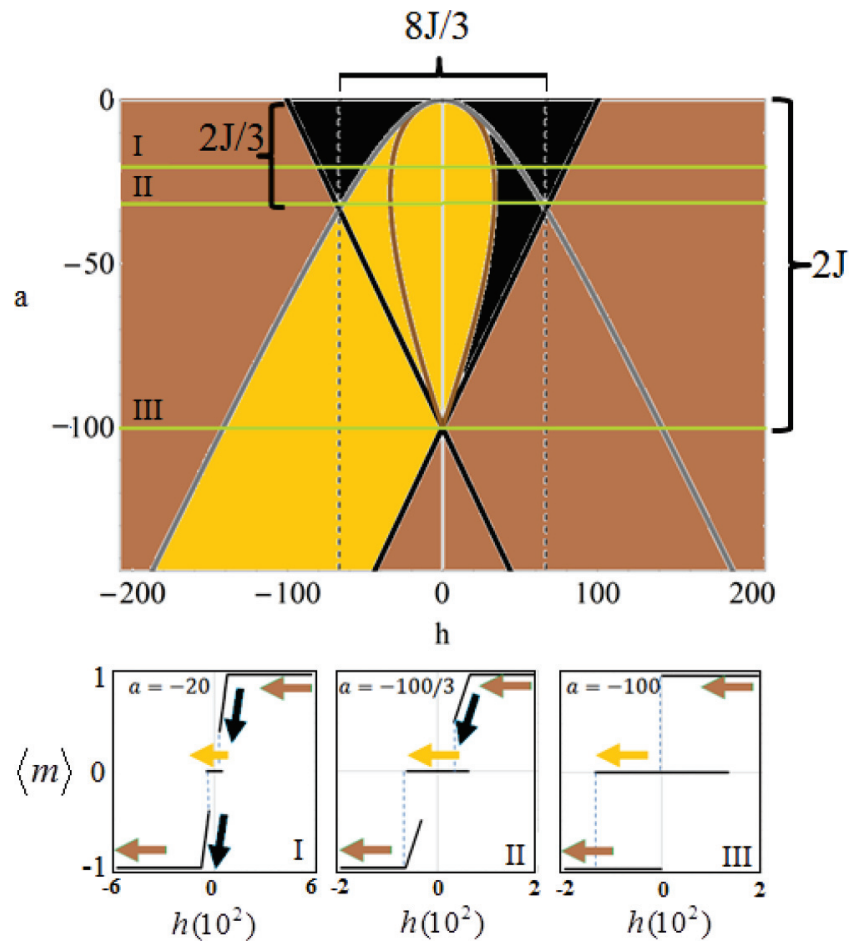

FIG. 1. (Color online) Phase diagrams of different states for two coupled nanomagnets arising in a magnetic field applied orthogonal to the easy $y-z$ plane, i.e., in the $x$ direction of the main axis of anisotropy. The anisotropy parameter $a$ depends on the aspect ratio of the nanomagnets and the saturation magnetization. The maximum critical field varies for different levels of anisotropy. The horizontal lines through the top $a$ - $h$ diagram, at locations I, II, and III, have associated hysteresis diagrams shown in the $\langle m\rangle-h$ plots. When $J=-50$ there are three different kinds of states, i.e., when the magnetization orientation is parallel (intermediate hue, brown online), scissoring (black), and antiparallel (light coloration, light orange online). Through line I the anisotropy is $a=-20$ and the transition from parallel alignment (brown online) to scissoring state (black online) occurs at $h=a-2 J=80$. This state persists until $h=-\left(2 a J-a^{2}\right) / \sqrt{2 a J+a^{2}}=32.66$ and the emergence of the antiparallel state (light orange online). The next transition is to another scissoring state at $h=-\sqrt{2 a J+a^{2}}=-49$. The negative saturation field occurs after this state and a parallel state occurs from $h=2 J-a=-80$ onwards. We call this regime, which exhibits these characteristics up to $a \leqslant 2 J / 3$, the $A F 1$ phase. Line II exhibits identical magnetization characteristics to line I, but it is the boundary between the $A F 1$ and $A F 2$ phases. The phase boundary is at $\{h, a\}=\{ \pm 4 J / 3,2 J / 3\}$. Line III marks another critical transition in the phase diagram at $a=2 J=-100$. This phase, classified as $A P$, is indicative of magnetization evolution states whereby $a \geqslant 2 J$ and there is no scissoring state.

lines of stability separating scissored states from antiparallel states (lightly colored areas-light orange online-in Fig. 1) are

$$
h_{\nearrow \searrow \rightleftharpoons \leftarrow \rightarrow}=-\frac{\left(2 a J-a^{2}\right)}{\sqrt{2 a J+a^{2}}}
$$

and

$$
h_{\swarrow \nwarrow \rightleftharpoons \leftarrow}=\frac{\left(2 a J-a^{2}\right)}{\sqrt{2 a J+a^{2}}} .
$$

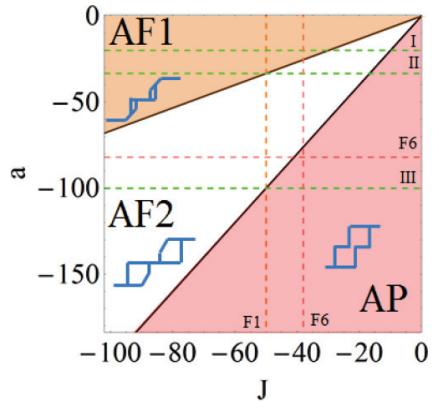

FIG. 2. (Color online) The phase diagram of three main antiferromagnetic phases of the two nanomagnets: $A F 1, A F 2$, and $A P$ is shown as a function of the antiferromagnetic coupling $J$ and $a$. The $A F 1$ phase occurs when $0<a \leqslant 2 J / 3$, whereas the $A F 2$ phase occurs as $2 J / 3<a \leqslant 2 J$. The AP phase exists when $a>2 J$. The boundaries between the phases are shown by the solid dark lines. The general shape of the total magnetization hysteresis as a function of the applied field is sketched within the zone of each phase. The dashed line (dark orange online) denoted $F 1$ is related to the value of $J=-50$ as used in Fig. 1. Also, the dashed lines (green online) marked I-III correspond with I-III in Fig. 1. Likewise, the dashed lines marked $F 6$ are for $J=-35$ and $a=-83$, which are the values used in Figs. 6-9.

When one solves the aforementioned determinant for $\varphi_{1}=\varphi_{2}$, one also finds for the transitions between antiparallel (lightly colored area in Fig. 1) and scissored states (black regions in Fig. 1)

$$
\begin{gathered}
h_{\leftarrow \rightarrow \rightleftharpoons \nwarrow}=-\sqrt{2 a J+a^{2}}, \\
h_{\leftarrow \rightarrow \rightleftharpoons \nearrow \searrow}=\sqrt{2 a J+a^{2}},
\end{gathered}
$$

under the condition that $a \geqslant 2 J / 3$. Also, transitions between the antiparallel states and parallel (intermediate hue, brown online, in Fig. 1) occur at

$$
\begin{aligned}
& h_{\leftarrow \rightarrow \rightleftharpoons \leftarrow \leftarrow}=-\sqrt{2 a J+a^{2}}, \\
& h_{\leftarrow \rightarrow \rightleftharpoons \rightarrow \rightarrow}=\sqrt{2 a J+a^{2}},
\end{aligned}
$$

under the stipulation that $a<2 J / 3$.

In Fig. 2, the phases of the nanomagnets are shown as a balance between the anisotropy parameter $a$ and coupling $J$. When $0<a \leqslant 2 J / 3$, the average magnetization, as a function of the applied magnetic field, is characterized by having a Barkhausen jump from an antiparallel state of the magnetic moments into a scissored state (spin-flop state). This characteristic is associated with the $A F 1$ phase. Between the limits of $2 J / 3<a \leqslant 2 J$ in Fig. 2, the average magnetization has the propensity to exhibit a Barkhausen jump from an antiparallel state directly into a parallel saturated state, without a transition through a scissoring state. This is the $A F 2$ phase. The typical form for $A F 2$ phase hysteresis is shown in Fig. 4.

The $A F 1$ and $A F 2$ phases have characteristic Barkhausen jumps from scissored states into antiparallel states [from point " $B$ " to point " $C$ " in Figs. 3(a) and 5(a)]. In the $A F 1$ phase there is a second-order state change from the scissored state into a parallel state that is marked by a smooth transition [at points $A$ and $F$ in Fig. 3(a)]. The $A F 2$ phase does not have a transition from a scissored state to the parallel state. 


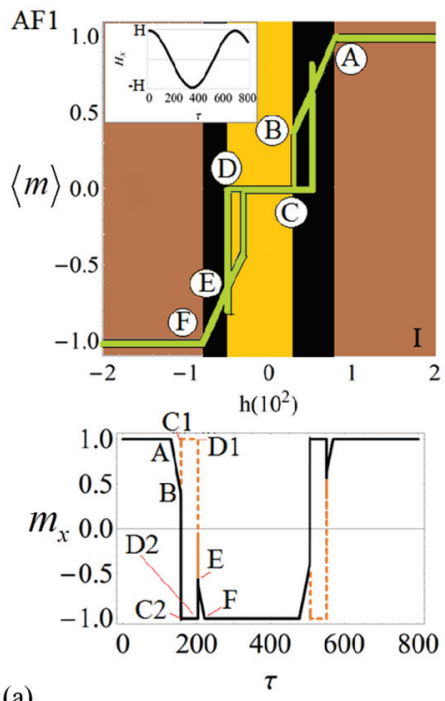

(a)

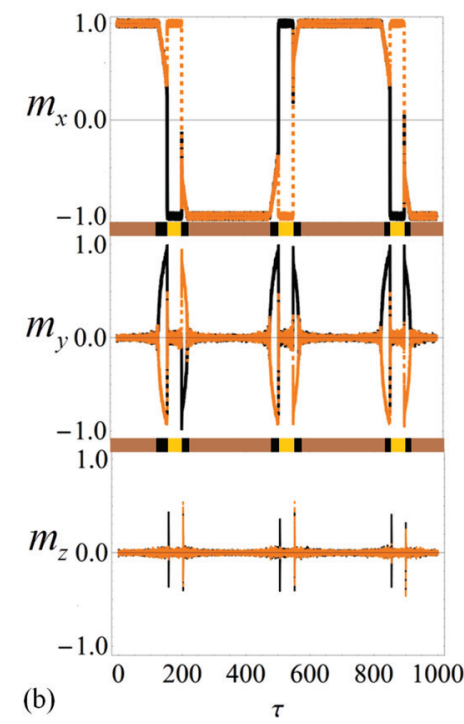

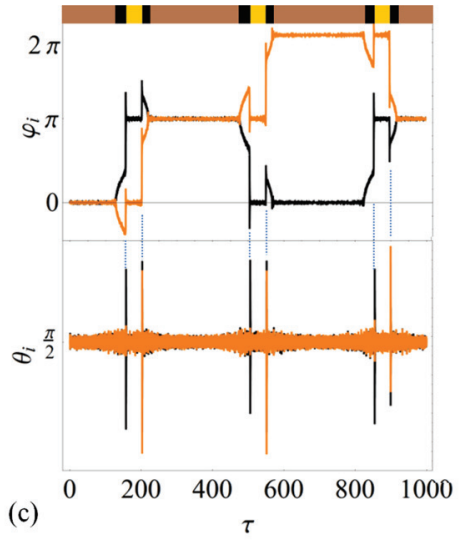

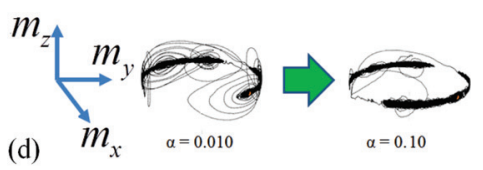

FIG. 3. (Color online) The AF1 phase for two nanomagnets with dimensions $l_{x}=41 \mathrm{~nm}, l_{y}=8 \mathrm{~nm}$, and $l_{z}=2 \mathrm{~nm}$. In dimensionless units the anisotropy parameters take the values $a=-19$ and $b=-54$, the coupling $-J=-50$, the frequency $-f=0.009$, and the damping parameter is small, $\alpha=0.01$ with $r=0.08$. (a) Top plot: the average total magnetization. The time evolution of the applied magnetic field $H_{x}$ is shown in the inset. (a) Bottom plot: the evolution of $m_{x}$ as a function of time $\tau$. Here dashed lines (dark orange) and solid lines (black) are for the first and second nanomagnet, respectively. There are four critical points of stability shown (see Ref. 25 for a comparison): $A, B, D$, and $F$. At point $A$ there is a transition from a parallel alignment to one of a scissored state (Ref. 30). At point $B$ a sudden switch into the purely antiparallel state is made (indicated by $C$ ). This is demonstrated in the bottom plot of (a) at points $C 1$ and $C 2$, i.e., $m_{x}=1$ and $m_{x}=-1$, respectively. Such AP state continues until $D$ when the coupled magnets reacquire a scissored state (the transition is marked by $D 1$ and $D 2$, and point $E$ marks the beginning of the new state). At $F, m_{x}$ in magnet " 1 " is the same as in magnet " 2 " and a parallel state occurs at negative magnetization saturation. (b) The magnetization components as a function of time, $\tau$. In (c) one can see the polar angles $\theta_{i}$ and azimuthal angles $\varphi_{i}$ characterizing the magnetization of the magnets. The gray coloration (dark orange online) represents the aforementioned angles [or magnetizations in (b)] for one nanomagnet and black for the other. The real time is given by $t=\left(\gamma M_{s}\right)^{-1} \tau$ and the saturation magnetization is $M_{s}=5 \times 10^{5} \mathrm{~A} / \mathrm{m}$. The same colors, in (a)-(c), and in Fig. 1 in the background shading for the nanomagnets states: parallel (intermediate hue, brown online), scissoring (black), and antiparallel (light coloration, light orange online), are used. In (d) as damping increases from $\alpha=0.01$ to $\alpha=0.1$ (and $r=0.22$ ), the amplitude of $m_{z}$ oscillations reduces.

However, both $A F 1$ and $A F 2$ phases have transitions from parallel states into scissored states [the point "A" in Figs. 3(a) and 5(a)]. The final phase associated with antiferromagnetic coupling, designated the notation $A P$, only has antiparallel states and parallel states and there is no development of a scissored state in the hysteresis profile. Thus, we have a generic description of nanomagnets of different material properties, dimensions, and coupling interactions which is particularly useful for doped $\mathrm{CoFeB}$ compounds due to their amenable saturation magnetization characteristics. These phases are relatively stable for levels of damping that are associated with $\mathrm{CoFeB}$ materials $(\approx \alpha=0.01)$.

\section{COMPARISON OF THE QUASISTATIC AND DYNAMICAL RESULTS}

The validity of the quasistatic approximation to understand the hysteresis characteristics of coupled nanomagnets will be shown in the following details to be excellent and complementary to a full dynamical investigation. The phase diagram shown for the quasistatic results, Fig. 1, indicates the basic character of the dynamical hysteresis too. However, the dynamical analysis reveals transitory oscillatory behavior in the magnetization switching, whereas in the quasistatic analysis magnetization changes, at the critical points of stability, are simply seen as spontaneous jumps between states. In this analysis we show that the quasistatic approximation allows one to predict the properties of coupled nanomagnet hysteresis even for reasonably small levels of Gilbert damping. On account of the small thickness of the nanomagnets and their elongated shape, a strong $z$-axis anisotropy exists that makes the quasistatic approximation reasonable. In the dynamical analysis the $z$-axis anisotropy is made the largest, which corresponds to $b \gg a$, throughout the examples in this work. In each of the figures, the hysteresis and time evolution of $m_{x 1}$ and $m_{x 2}$ are illustrated. For convenience, the hysteresis is given in terms of the average combined magnetization of the two nanomagnets in the SAF structure,

$$
\langle m\rangle=\left(\cos \varphi_{1} \sin \theta_{1}+\cos \varphi_{2} \sin \theta_{2}\right) / 2 .
$$

With the aim to reproduce the quasistatic hysteresis characteristics shown in Fig. 1, a small oscillatory fluctuation in the magnetic field is implemented in the dynamical analysis. The requirement for this is because the noise can result in the creation of more switching pathways by driving the system into adjacent metastable states. We introduce stochastic dynamics into the model of the two nanomagnets by the inclusion of a stochastic, thermally induced magnetic field fluctuation. A different magnetic field fluctuation exists for each nanomagnet as the thermal fluctuations in each nanomagnet are regarded 
as uncorrelated. ${ }^{20}$ To include thermal fluctuations into the analysis we add a thermal field, $\mathbf{H}_{\text {therm, }}$ to Eq. (4). The introduction of this term allows the description of a thermally assisted magnetization reversal in an applied magnetic field. The dimensionless thermal field is written as

$$
H_{\text {therm }}(t)=\frac{1}{M_{s}} \sqrt{\frac{2 \alpha K_{B} T}{\mu_{0} V M_{s} \gamma \Delta t}} g(t),
$$

where $K_{B}$ is Boltzmann's constant, $T$ is the temperature of the thermal bath, and $\Delta t$ is a discretized time interval that is chosen to correspond to the switching time of the nanomagnets (typically $1-10 \mathrm{ps}$ ). The thermal field is described as a Gaussian random process ${ }^{21}$ including a Gaussian distribution with zero mean and unit variance. ${ }^{22}$ The random numbers $g(\tau)$ that are used are then generated through the use of the Box-Müller algorithm ${ }^{23}$ and vary between \pm 1 at each time step. The thermally induced field $h_{\text {therm }}$ (where the $H_{\text {therm }}$ is normalization by $N_{x}$ ), is introduced into the Landau-LifshitzGilbert equations alongside the applied magnetic field $h_{\text {app }, j}$,

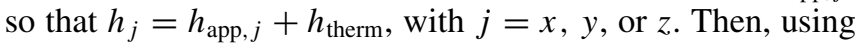
$\tau=\gamma M_{s}$, Eq. (15) becomes

$$
h_{\text {therm }}(\tau)=\sqrt{\frac{2 \alpha K_{B} T}{\mu_{0} V M_{s}^{2} N_{x}^{2} \gamma \Delta \tau}} g(\tau)=r g(\tau) .
$$

The amount of thermally activated magnetic noise follows from the fluctuation dissipation theorem ${ }^{24}$ that considers the effects of the thermal environment, thermal fluctuations, and energy dissipation as having the same origin. We write the strength of the thermal magnetic field in terms of $r$, as defined in Eq. (16). Thus, a random magnetic field is added to the $h_{x, y, z}$ components. If there is no perturbation, the moments of the two nanomagnets move very nearly symmetrically and the phases associated with the quasistatic approximation for a SAF generally do not appear. Noise in the system is necessary to drive the system into the metastable states. The thermal excitation can help the system to leave local energy minima (metastable states) and reach the global minimum. This is an example of temperature-influenced development, as the temperature has no visible effect when the system has obtained a deep energy minimum and therefore behaves statically. The random temperature fluctuations have great influence when the system evolves rapidly and a high degree of sensitivity to external influences emerges. Thus, in using Eqs. (15) and (16) we gain quantitative and qualitative understanding of the phases of different nanomagnetic systems that are influenced by thermal noise and the phase locking and unlocking of these coupled nanomagnets. To see the critical points of stability ${ }^{25}$ it is useful at times to take a more qualitative perspective and this can be done by reducing the thermal noise to a level where the noise-induced oscillations are minimal. In Fig. 3, the dynamical $A F 1$ phase is shown for $a=-19$, with the fluctuations defined by Eq. (16). Here $r=0.08$ and the Gilbert damping is $\alpha=0.01$. It is exactly as one would expect from the phase diagram of Fig. 1. There are positive and negative saturated parallel states where the moments are aligned and there is a scissored state which is defined by Eq. (9). The magnetic field at which the system leaves the antiparallel state is smaller than $a-2 J$ and as a consequence the scissored state occurs both when the field is reduced from saturation towards zero and when the field is increased from zero to saturation. The $A F 1$ phase is better known as the spin-flop mode in the literature, after Worledge ${ }^{7}$ first made the quasistatic phase diagram of $h-J$ characteristics for two identical coupled nanomagnets. In the $A F 1$ phase in Fig. 3 with $\alpha=0.01$, the value of magnetic field required to switch the coupled nanomagnets magnetizations from one parallel state to the other is $27 \mathrm{kOe}(h=160)$, whereas the switching between an antiparallel state into a scissored state and back occurs over a range of $3700 \mathrm{Oe}(h=22)$. One can see in Fig. 3(b), that the $m_{x}, m_{y}$, and $m_{z}$ components of the magnetizations in each magnet rapidly switch near critical points of stability, most notably in the transitions to/from a scissored state. In Fig. 3(c), one can see these transitions as the rapid rotation of the azimuthal angles $\varphi_{i}$ in the lead-up to the Barkhausen jumps. Later, in Fig. 11 these switching events are looked at in more detail for the $A F 1$ phase. The $A F 1$ phase has been found experimentally in a number of different $\mathrm{SAF}$ structures, ranging from $\mathrm{NiFe} / \mathrm{Ru} / \mathrm{NiFe}$ (Ref. 26) to $\mathrm{CoFeB} / \mathrm{Ru} / \mathrm{CoFeB} .{ }^{27}$ The exchange bias effect in ferromagnetically/antiferromagnetically coupled stacks of nanomagnets can be used to useful effect to control the magnetic coercivity and the critical field levels before the onset of Barkhausen jumps. The interface morphology and system temperature can control the phase of the SAF structure and entice a transition from phase $A F 1$ to $A F 2$, i.e., by an increase in the maximum magnetic field strength of the antiparallel state. The magnetic ordering in the magnetic multilayers is controlled by the exchange energy as the magnetizations of the two ferromagnetic layers are coupled by the electrons in the spacer layer. The sign of the coupling oscillates as a function of the interlayer thickness. In this work the magnetizations of the two layers are usually antiparallel when there is no applied magnetic field because they are taken to be antiferromagnetically coupled. The application of the external field overcomes this coupling and brings the magnetizations of the two layers into parallel alignment at a saturating field strength. This change in the orientation of the magnetizations leads to a change in resistance and gives rise to the giantmagnetoresistance effect. ${ }^{28,29}$ The interface between the two nanomagnets behaves as a potential step for the electrons. The electrons that strike it have a transmission probability that falls from one. An interpretation of the mechanism of exchange interaction can be given by quantum interference effects, which occur as the Bloch waves at the interfaces between the normal metal and the ferromagnetic layers reflect in the confined geometry of the spacer layer. These spindependent reflections and the electron-spin motion give an analogy to the optical Fabry-Perot resonator and a quantum barrier description. ${ }^{31,32}$ A temperature driven reorientation transition can be induced as the temperature increases and thus a phase change can occur. There is an important role for the magnetic anisotropy in connection with the temperature dependence of the interlayer coupling in the $A F 1$ and $A F 2$ phases. As pointed out in Ref. 27, as temperature increases, the antiferromagnetic coupling is overcome by thermal activation. Interlayer exchange coupling, magnetic anisotropy, and the balance with temperature and an applied field or current play the most important roles for sensor applications using the giant-magnetoresistance effect. 


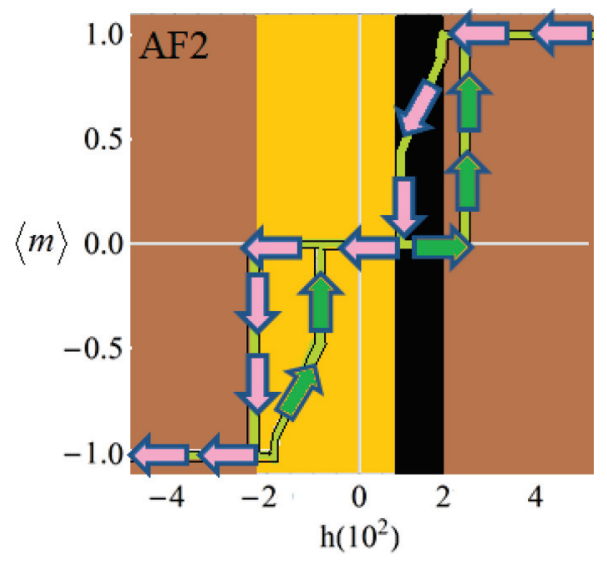

FIG. 4. (Color online) A typical $A F 2$ phase hysteresis diagram. The maximum magnetic field (see the background shading as in Fig. 1) at which an antiparallel state exists (light orange online) extends into a well-established level of the parallel state, which is beyond the level marking the onset of the saturation field.

For clarity, the typical form for the static or dynamical $A F 2$ phase hysteresis is shown in Fig. 4, where one can see that the maximum magnetic field associated with antiparallel alignment of the magnetizations $\left(H_{\max }^{A P}\right)$ before a Barkhausen jump occurs, has extended (as compared to the $A F 1$ phase). Whenever the system resides in a purely antiparallel state, upon an increase or decrease in magnetic field strength the result is a rapid transition to the parallel state when $H_{\max }^{A P}$ is reached. The dynamical $A F 2$ phase is valid for thin elongated SAF within the range $2 J / 3 \leqslant a<2 J$. The boundary between the $A F 1$ and $A F 2$ phases is illustrated at $a=2 J / 3$ in Fig. 1. In Fig. 1 the transition hysteresis loop between the $A F 1$ and $A F 2$ phases is shown with $J=-50$ and $a=-100 / 3$. The comparison to the dynamical hysteresis for $J=-50$ and $a=$ $-100 / 3$ is illustrated in Fig. 5(a) and is seen to have very good agreement. In this figure the transition between the fully parallel state (at point $A$, where $h \approx 70$ ) and an antiparallel state (at point $C$, where $h \approx 30$ ) happens through an intermediary scissored to antiparallel state transition (at point $B$, where $h \approx$ 30). This develops over a range of 3010 Oe $(h=36)$. When the anisotropy parameter $a>2 J$, then the $A P$ phase develops.

Typical hysteresis curves for the $A P$ phase are shown in Figs. 6-8 for average magnetization, Eq. (14), as a function of the magnetic field strength for different damping strengths. In the $A P$ phase, when starting from a level of saturated magnetic field and lowering the field in accordance with Eq. (6), the magnetization states of the nanomagnets change from parallel to antiparallel as the $H_{\max }^{A P}$ magnetic field strength is approached. However, damping and thermal fluctuations exert persuasive control over the magnetization switching, and can strongly influence the characteristics of the system, thus changing the phase from $A P$ to $P$. The propensity for this to happen is illustrated in the sequence of figures, Figs. 6-9, where the nature of the system changes in response to the strength of the damping and consequently the level of the thermal magnetic field. The result of temperature fluctuations can be a switching field also of a fluctuating nature during a reentrant magnetization process. ${ }^{33,34}$ In Figs. 6 and 7 (both of which are for nanomagnets with $J=-35, a=-83$, and
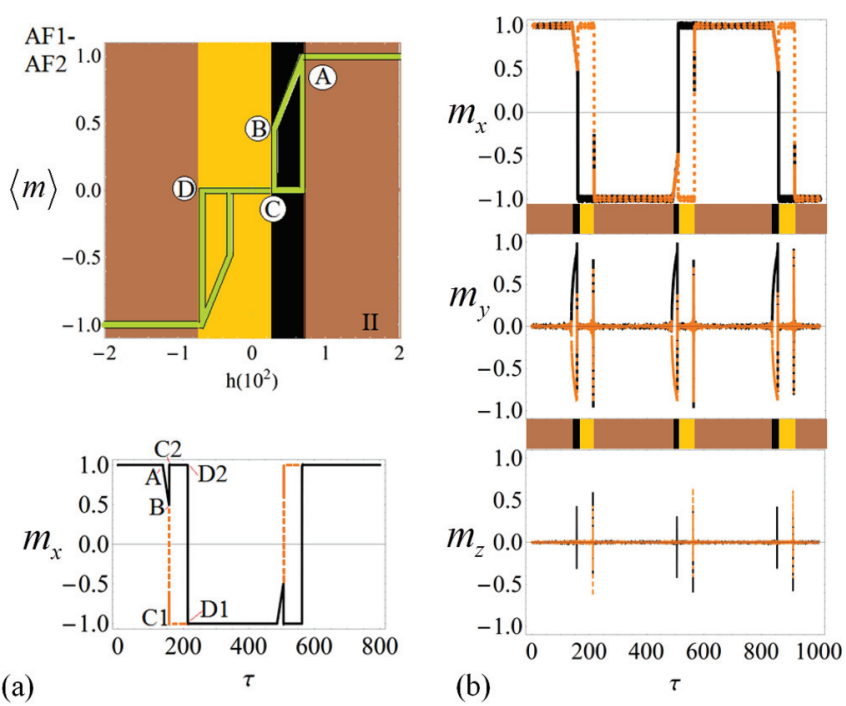

(a)

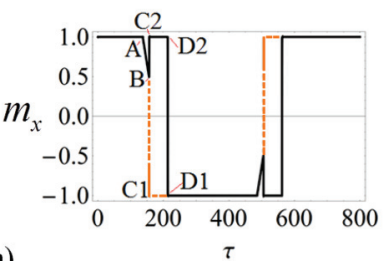

FIG. 5. (Color online) (a) Top plot: the total magnetization of the two nanomagnets at the $A F 1-A F 2$ transition. This hysteresis is right at the phase boundary. The background color shading is the same as in Fig. 1. At the bottom plot is the evolution of $m_{x}$ as a function of time $\tau$. The dashed and solid lines are for the first and second nanomagnets, respectively, which have dimensions $l_{x}=75 \mathrm{~nm}, l_{y}=10 \mathrm{~nm}$, and $l_{z}=2 \mathrm{~nm}$, the saturation magnetization $M_{s}=5 \times 10^{5} \mathrm{~A} / \mathrm{m}, J=-50, a=-100 / 3, b=-115, r=0.1$. The Gilbert damping and the frequency are small, $\alpha=0.01$, and $f=0.009$, respectively. There are three critical points of stability (see Ref. 25 for a comparison): $A, B$, and $D$ when the field changes. At point $A$ the magnetic moments of the two magnets make the transition from a parallel to a scissored state (Ref. 30). The temporal evolution of the magnetic moments proceeds in this state until $B$, at which point there is a switch to the antiparallel state. At $D$ a rapid switch from the antiparallel to parallel alignment occurs and the magnetizations saturate to the opposite polarity to which they were in preceding $A$. In (b) we show the evolution of the magnetization components $m_{x, y, z}$ as a function of time $\tau$.

$b=-614)$, one can also see a comparison between the effects that are attributable to moderate and weak damping, respectively. When there is little damping exerted on the system, the energy transport of the magnetization is largely conservative and with the influence of a small thermal fluctuation the entropy of the system becomes higher and the random transitions between energy minima are more prevalent. The low damping scenario, which includes thermal fluctuations, gives a different nature to the $A P$ phase to that of a relatively higher damping: In the hysteresis of Fig. 6(a) the system allows the direct transition between completely parallel magnetization states of different polarity, whereas with larger damping an indirect transition via an antiparallel state can also happen. In Fig. 6(a) one can see two different kinds of transition between parallel states $(\rightarrow \rightarrow$ to $\leftarrow \leftarrow$, and vice versa). One of them is an indirect transition, which means that the system first changes almost spontaneously to an antiparallel state at about $H_{x}=-150 \mathrm{Oe}$ and then later transitions to the opposite parallel state at -2150 Oe. This is shown in Fig. 6 for the first cycle of the applied magnetic field. 
(a)

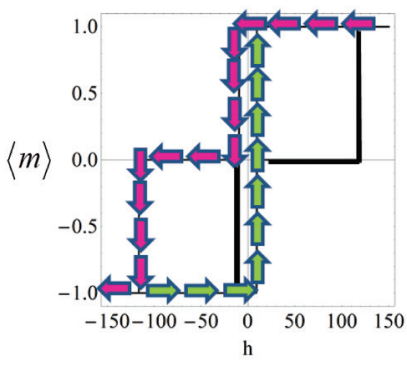

(b)

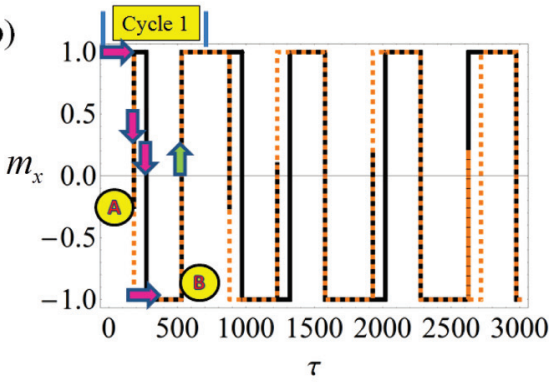

(c)

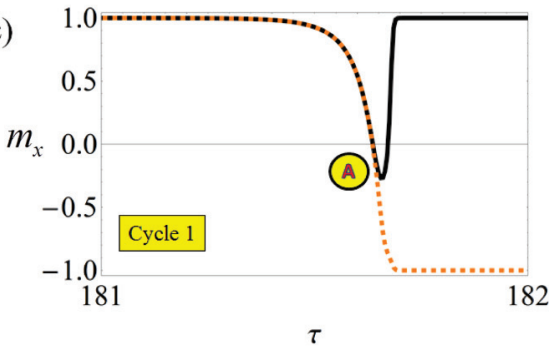

(d)

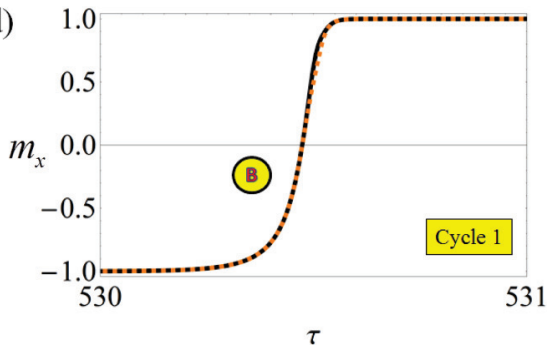

FIG. 6. (Color online) (a) The $A P$ to $P$ hysteresis evolution over one cycle of the applied magnetic field is mapped by the arrows around the $\langle m\rangle$ against $h$ plot in the $A P$ phase existing for parameters $J=-35, a=-83$, and $b=-614$. The sizes of two identical nanomagnets are $l_{x}=325 \mathrm{~nm}, l_{y}=25 \mathrm{~nm}$, and $l_{z}=2 \mathrm{~nm}$. The thermal field is $r=0.134$ with a Gilbert damping $\alpha=0.01$. Further cycles of the applied magnetic field bring about the emergence of different paths around the hysteresis loop (as indicated by the black lines). Transitions between the parallel states $\leftarrow \leftarrow \rightleftharpoons \rightarrow \rightarrow$ are also very likely to occur, as is the cycle of $\rightarrow \rightarrow \rightleftharpoons \leftarrow \rightarrow \rightleftharpoons \leftarrow \leftarrow$, $\rightleftharpoons \leftarrow \rightarrow \rightleftharpoons \rightarrow$, i.e., a symmetric hysteresis cycle inclusive of antiparallel and parallel states. (b) The $m_{x}$ component in each of the two nanomagnets [the first magnets magnetization evolution shown in black lines and the second by dashed gray lines (dark orange online)] as a function of time $\tau$. In the first cycle of the time dependent applied magnetic field " $A$ " and " $B$ " mark transition points between states. Magnified images of these transitions in time are shown in (c) and (d).

One notes that when the damping is $\alpha \leqslant 0.04$, as in the case of Fig. 6, that all transitions between the antiparallel and parallel and oppositely polarized parallel states are possible. Below $\alpha=0.04$ the system is characterized by a higher
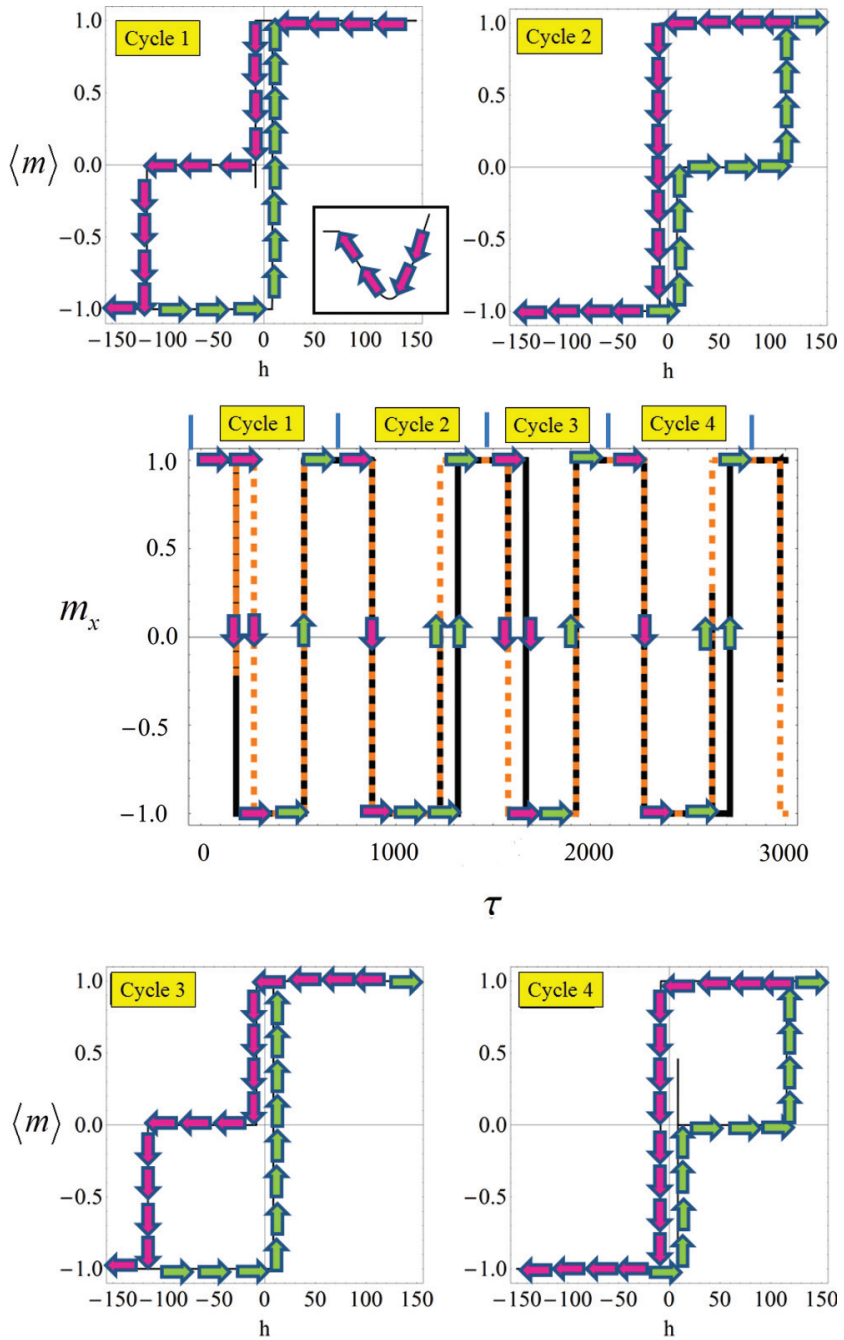

FIG. 7. (Color online) The same parameters as in Fig. 6 except the damping increases, $\alpha=0.04$, and the thermal field strength is 0.02. Here four cycles of the applied magnetic field are performed, each showing the asymmetric hysteresis curve. One should note that due to the random nature of the system, direct paths between the parallel states do also occur as time goes on, as in Fig. 6, but less frequently for these values of $\alpha$ and $r$. More likely than direct transitions between parallel states, is the emergence of intermediary antiparallel states. One can see in the inset of the top left hysteresis curve a magnification of the small oscillation in the magnetization as the transition from $\langle m\rangle=1$ to $\langle m\rangle=0$ occurs. The system had begun the transition to the $\langle m\rangle=-1$ state but the energy to do so, including the random noise, was not quite high enough on that occasion.

stochastic nature, whereby in each cycle of the applied magnetic field, any possible path between the existing energy minima is demonstrated. Only for very low thermal fields $(r \approx 0.002)$ does the regular stable hysteresis cycle of Fig. 7 occur for $\alpha<0.04$. For the nanomagnetic system described with an $A P$ phase in Figs. 6-9, when $\alpha \geqslant 0.04$ the pattern of hysteresis becomes more predictable even against thermal fluctuations. Hence, the black lines in Fig. 6(a) in the $\langle m\rangle$ versus $h$ plot are drawn to chart all the possible paths of the hysteresis as the number of cycles increases. When the magnetic field is increasing from negative $h$ and $\langle m\rangle=-1$, in Fig. 6(a), the next transition between parallel states is seen to 
(a)
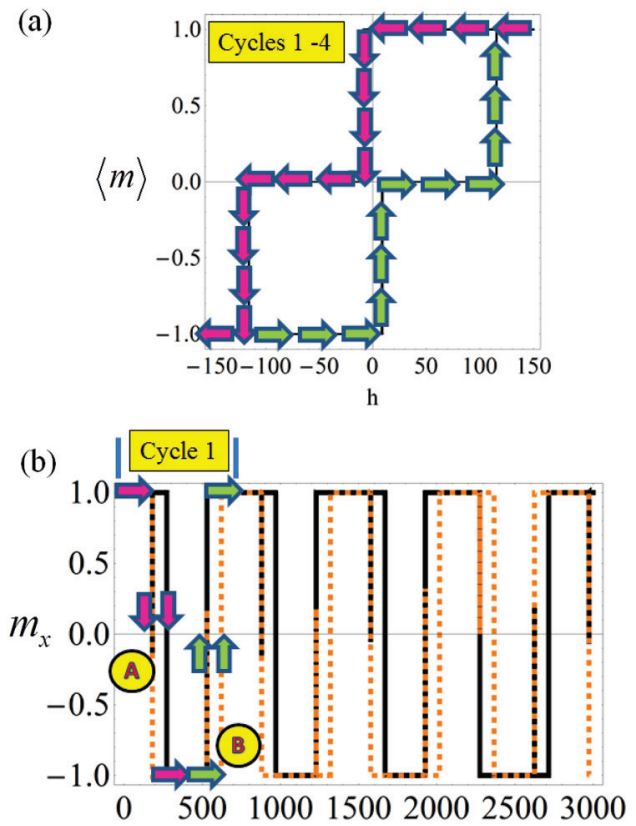

(c)

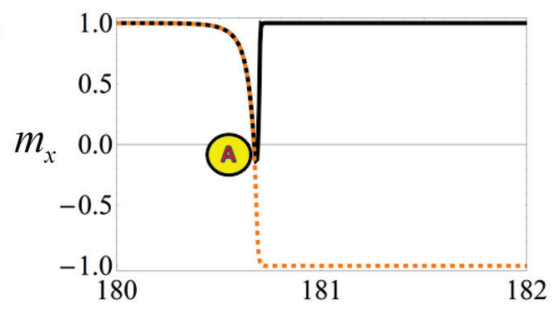

(d)

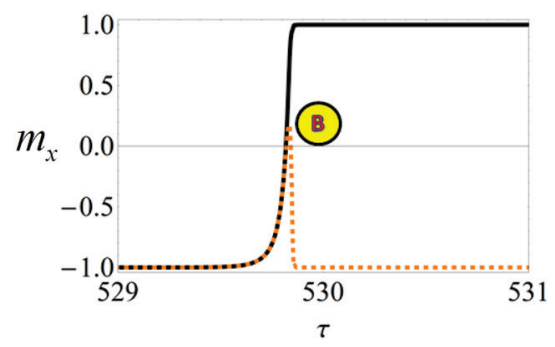

FIG. 8. (Color online) The same parameters are used as in Fig. 6, other than the adjustment of the damping and thermal field to $\alpha=$ 0.04 and $r=0.37$. In this situation, the hysteresis curve in (a) always takes the form shown. (b) The dependence of the $m_{x}$ component of the magnetization on time $\tau$, for the first four cycles of the ac applied magnetic field. The first cycle transitions from parallel to antiparallel states are shown in closer detail in (c) and (d). The real time is found from $t=\left(\gamma M_{s}\right)^{-1} \tau$.

be direct, i.e., the system by-passes the antiparallel state and rapidly moves to the $\rightarrow \rightarrow$ state at $\approx 150$ Oe. This inevitably leads to much smaller switching fields and, in this example, on the return loop of the hysteresis a transition between parallel states can happen through a change in applied magnetic field strength of 210 Oe. In Fig. 7, a slight increase in the thermal magnetic field parameter $r$ to 0.02 and an increase in the damping to $\alpha=0.04$, as compared to the values in Fig. 6, results in a greater likelihood for the asymmetric hysteresis cycle discussed for Fig. 6 to prevail. Indeed, it occurs in every cycle of the particular example given in Fig. 7 as the time (a)
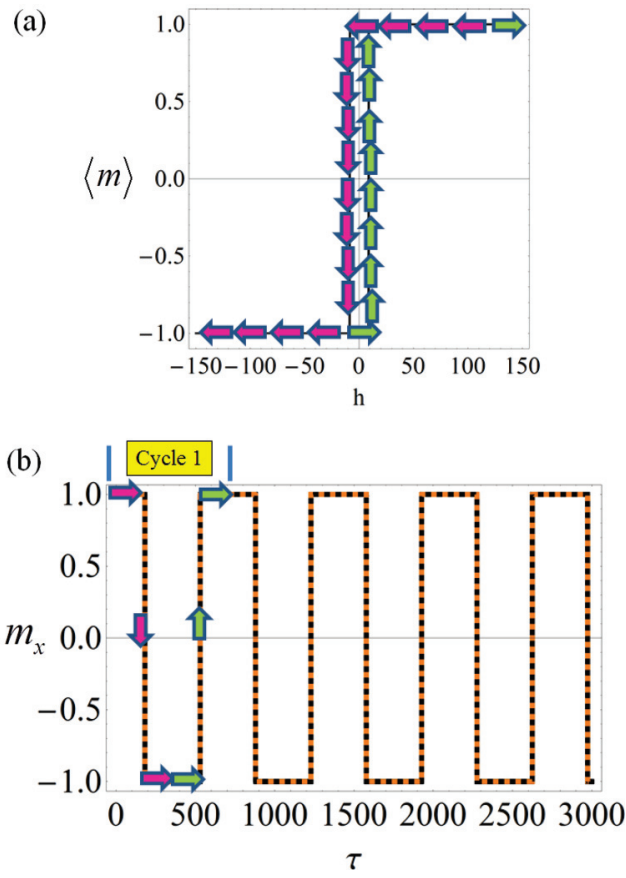

FIG. 9. (Color online) The same parameters are used as in Fig. 6, except $\alpha=0.04$ and $r=0.002$. The rectangular hysteresis loop dominates throughout the evolution of $\langle m\rangle$ with time $\tau$. This is the same hysteresis profile as in a single elliptical nanomagnet that is subjected to an applied magnetic field along its longest dimension. The difference is that the coupling strength $J$ can alter the width of the hysteresis path, i.e., increase or decrease the coercivity. Here $J=-35$ and if $J>-35$, the coercivity increases.

dependent applied magnetic field cycles through positive and negative values. One sees a rectangular hysteresis path in the $\langle m\rangle$ versus $h$ plots less frequently in using the parameters of $\alpha$ and $r$ of Fig. 7 than Fig. 6, but still often enough to categorize this evolution of $\langle m\rangle$ as the same subspecies of the $A P$ phase. In Fig. 8, where $\alpha=0.04$ and there is a much larger value of $r=0.37$, there are consistently state transitions that must go from a parallel state into an antiparallel state to find the oppositely polarized parallel state. In Fig. 9 a fully parallel phase can be seen to have emerged when the system, with the same sized nanomagnets as in Figs. 6-8, has values $\alpha=0.04$ and $r=0.002$. Here, there is no transition to an antiparallel state. The whole hysteresis profile occurs over 380 Oe. This would imply the desirability of obtaining the parallel phase when designing the nanomagnets for logic and memory applications due to the small switching fields that can occur. To increase the coercivity of the hysteresis profile in Fig. 9 the coupling strength $|J|$ needs to decrease. Metastability occurs in the energy landscape of the nanomagnets because local energy minima are separated by potential barriers that impede the immediate transition between them. A path is created through the state space that emerges so as to minimize the energy of the system. Upon reaching a localized minimum, then the tendency is for the system to relax into this state until some external perturbation, with enough energy, enables the transition to another local minimum. Thermal fluctuations can change the orientation of the magnetic moments of the system by providing enough stimulation for the barrier between local 

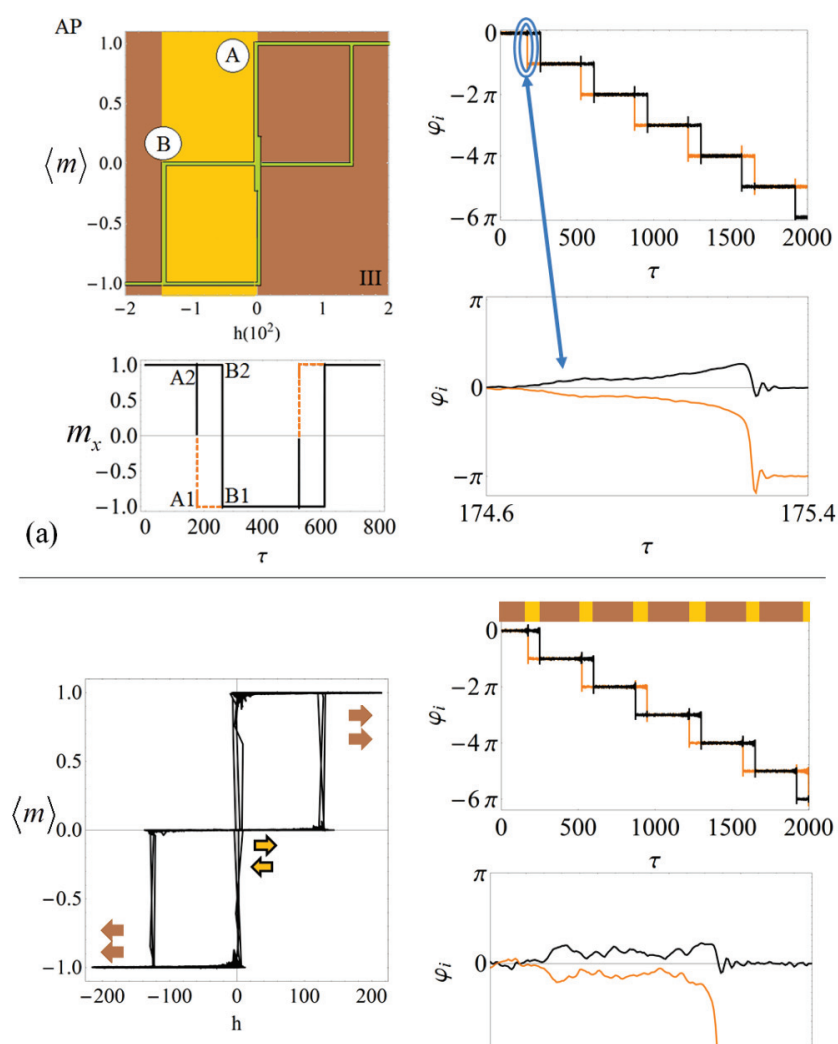

(b)

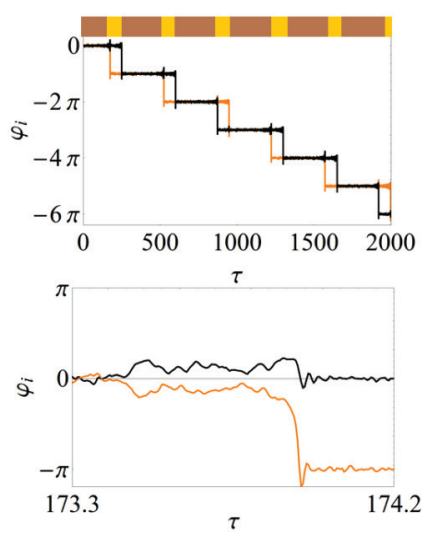

FIG. 10. (Color online) (a) The average combined magnetization of the two nanomagnets is shown on the top left plot. The evolution of $m_{x}$ as a function of time $\tau$ is given on the bottom left plot. The dashed (orange online) and solid black lines are for the first and second nanomagnets, respectively. The background shading is as in Fig. 1 . The nanomagnets dimensions are $l_{x}=155 \mathrm{~nm}, l_{y}=10 \mathrm{~nm}$, and $l_{z}=2 \mathrm{~nm}$. The saturation magnetization is $M_{s}=5 \times 10^{5} \mathrm{~A} / \mathrm{m}$. The Gilbert damping is $\alpha=0.002$ with $r=0.088$. In both plots $J=-50, a=-101, b=-345$, and $f=0.009$. There are two critical points of stability (see Ref. 25 for a comparison), $A$ and $B$. At point $A$ the magnetic moments configuration changes from parallel to antiparallel alignment. The temporal evolution of the magnetic moments proceeds in this state until $B$, at which point there is a switch to the parallel state. (b) The larger thermal field, $r=0.95$. The general shape of the hysteresis remains with some reentrant behavior (Ref. 33). The evolution of the azimuthal angles $\varphi_{i}$ in each nanomagnet is shown in both (a) and (b). A magnified view of the first switching from parallel to antiparallel state is given in the bottom right plots of (a) and (b).

minima to be crossed and when this happens the transition is very rapid. The reduction of an energy barrier height occurs when the system approaches a critical bifurcation point ${ }^{25}$ where a metastable state disappears. In Fig. 10(a), the onset of the $A P$ phase is shown, for a SAF system with $J=-50$ and $a=-101$, as predicted by the quasistatic phase diagrams. The transition between parallel states occurs with a switching field of 3970 Oe from point $A$ to the vicinity of point $B$ in Fig. 10. However, the transition between the parallel and antiparallel states occurs very near to $h=0$ and takes a magnetic field increase of 55 Oe to conclude. Thus, for logic or memory devices a small permanent static magnetic field could be used to hold the magnetizations in an antiparallel alignment (e.g., a "1" state). The application of a very small amplitude of oscillating or stepped magnetic field could then provide the impetus to switch to the parallel state ("0" state). Other magnetic states require much larger magnetic fields to be reached, so in a well shielded environment this may produce a low-field controlled memory element. In the $A P$ phase, starting from the saturated state, the two moments move symmetrically into opposite directions, cross the anisotropy energy barrier at the same time, and arrive in a local energy minimum after some decreasing oscillations. If there is no perturbation/temperature fluctuation $(r=0)$, none of the moments have enough energy to cross the energy barrier again, even if the transition would mean to proceed toward the global energy minimum. To reach this global energy minimum, a breaking of symmetry is necessary because in this situation only one of the moments should cross the barrier. The larger the additional energy (which is different for each individual nanomagnet) is, the higher the probability of reaching this state becomes. We emphasize the random nature of this movement. In Figs. 6-9 one can see an example of when the very same system can go to the antiparallel state at the first part of the hysteresis loop, but cannot go there at the second part of the loop, which without thermal fluctuations should just be the mirror image of the first part. The energy balance is subtle, but with higher values of $r$ (the fluctuation parameter) there is more likelihood for antiparallel states to appear (as in Fig. 8). With smaller values of $r$, and high $a / J$ ratios, the probability of the exclusion of the antiparallel state increases (see Fig. 9).

\section{THE DYNAMICAL SWITCHING OF MONODOMAIN NANOMAGNETS}

The understanding of switching times in artificially patterned arrays of nanomagnets has largely been derived from experiments on layers of two and three coupled nanomagnets ${ }^{35}$ or large arrays of patterned samples. ${ }^{36}$ These developments have occurred to meet the needs of the data storage industry. However, there is much still to be learned about even the most simplistic of SAF structures. Due to the transfer of energy occurring via complicated channels such as dipole-dipole and exchange coupling, damping, and anisotropic energies, the design of nanomagnetic devices is by no means straightforward. Doping amorphous materials such as $\mathrm{CoFeB}$ allows the opportunity to manipulate the saturation magnetization ${ }^{37}$ and consequently the damping of the system. The use of amorphous materials allows one to circumvent some of the design issues associated with crystalline magnetic structures, most notably strong crystalline anisotropy. Currently, however, the material most widely used in the industry is polycrystalline permalloy in which damping parameters as low as $\alpha=0.008$ have been found. ${ }^{38}$ The development of ultrafast magnetization dynamics is an extremely active research field, with subpicosecond switching on the horizon (e.g., see, Refs. 39 and 40). In all incarnations of rapid dynamical switching devices, the ferromagnetic relaxation aligns the magnetization with the applied field. The relaxation rate is intrinsic to a given material and is given by $G=\gamma M_{s} \alpha{ }^{41}$ For a material with $M_{s}=0.5 \mathrm{MA} / \mathrm{m}$ and $\alpha=0.01, G=1.1 \mathrm{GHz}$. In Fig. 11, an example of the evolution of the magnetizations of an $A F 1$ 

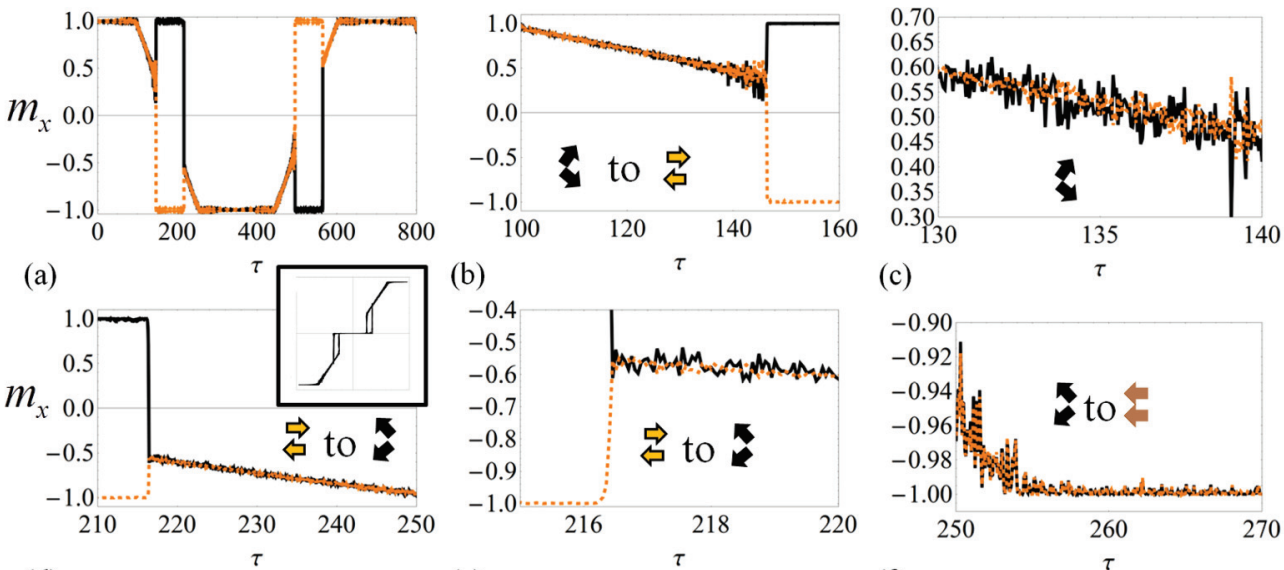

(c)

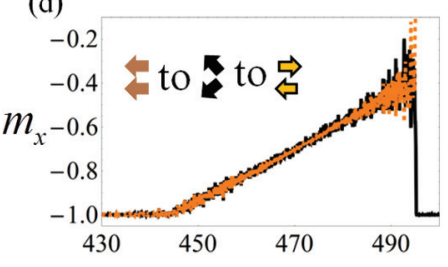

(e)

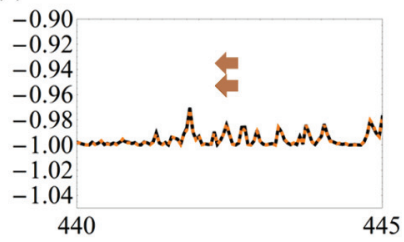

(g)

(h)
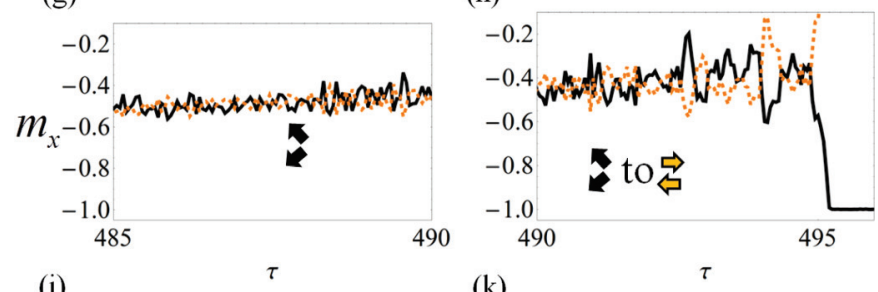

(k)

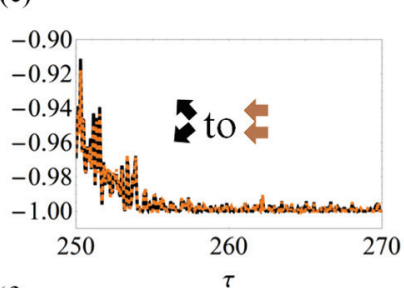

(f)

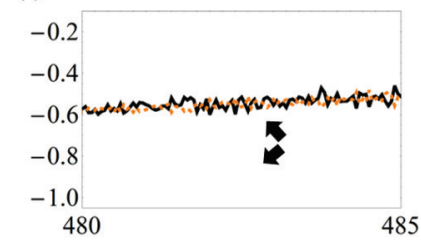

(i)

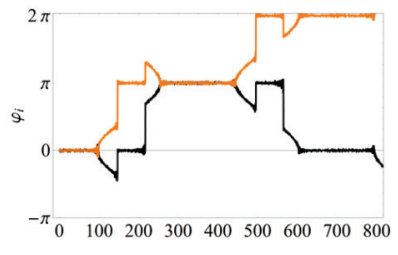

(1)

FIG. 11. (Color online) The switching times in the $A F 1$ phase between scissored and antiparallel states. The nanomagnets are taken to have dimensions $l_{x}=68 \mathrm{~nm}, l_{y}=10 \mathrm{~nm}$, and $l_{z}=2 \mathrm{~nm}$. The saturation magnetization is $M_{s}=5 \times 10^{5} \mathrm{~A} / \mathrm{m}$, the damping is $\alpha=0.01$, and $r=0.086$. The dashed lines show the character of $m_{x}$ in the first nanomagnet and the solid lines that of the second magnet. The real time is given by $t=\left(\gamma M_{s}\right)^{-1} \tau$. One can see that as the system moves towards a transition from a scissored to a parallel state the nanomagnets begin to synchronize [see (d) and (e), for example]. It is in these parallel states where $\langle m\rangle= \pm 1$ that phase locking can emerge when the system is in the right balance. The images in (b)-(k) are snapshots of that shown in (a). In (1) the switching of $\varphi_{i}$ as a function of $\tau=0-800$ is shown.

phase is given when the nanomagnets are subjected to the ac magnetic field with a frequency of $1 \mathrm{GHz}$. In all the following switching time descriptions, the time is measured from the onset of a transition to/from a parallel, scissored, or antiparallel state into a different state. Figure 11(a) shows the switching profile, $m_{x i}$ against $\tau$, for a whole hysteresis cycle of $m_{x i}$ for nanomagnets with $J=-80, a=-29, b=-99, \alpha=0.01$, and $r=0.086$. The magnetization flips from one parallel state to the other, $\rightarrow \rightarrow$ to $\leftarrow \leftarrow$, in $0.8 \mathrm{~ns}$. In the intermediate time between the emergence of the fully parallel states, there are scissoring state transitions that lead to/from antiparallel states. These are shown within the time evolution of $m_{x}$ in Fig. 11(b)-11(k) and will typically happen in 5 ps $(\nearrow \searrow$ to $\leftarrow \rightarrow$ ) and 3 ps $(\leftarrow \rightarrow$ to $\swarrow \nwarrow)$, respectively.

Likewise, in Fig. 12 the time evolution of $m_{x i}$ is shown for an $A F 2$ phase. In Fig. 12(a) the overall $\rightarrow \rightarrow$ to $\leftarrow \leftarrow$ switching occurs in $0.7 \mathrm{~ns}$ over a range of 9500 Oe. In Fig. 12(b), $\nearrow \searrow$ to $\longleftrightarrow \rightarrow$ takes place in 0.9 ps and in (c) $\leftarrow \rightarrow$ to $\swarrow \nwarrow$ occurs over 1.8 ps. In Fig. 12, the dimensionless parameters are $J=-50, a=-35, b=-164$, and $r=0.095$. The switching field to go from $\longleftrightarrow \rightarrow$ to $\rightarrow \rightarrow$ is $h=77$ (measured from $h=0$ ), which equates to 4750 Oe. Also, to return from $\rightarrow \rightarrow$ to $\leftarrow \rightarrow$ occurs in a change of field of $h=47 \equiv 2900$ Oe (see Fig. 4 for a similar hysteresis profile). In the $A F 2$ phase there are two main hysteresis loops, containing antiparallel, parallel, and scissoring states. For example, in Fig. 12 this hysteresis occurs between $0<\tau<220$ and translates as happening between $h=30$ and $h=58$ (range of 1730 Oe). Designing the magnetic device to operate in this regime would reduce the switching time and also the switching field required to polarize the parallel states. In the $A P$ phase, the switching times are around 2 ps for the example of Fig. $8: \rightarrow \rightarrow$ to $\leftarrow \rightarrow$ $(J=-35, a=-83, b=-614, \alpha=0.04$, and $r=0.37)$. The reliability of repeatable magnetic switching becomes less as the frequency increases. However, by carefully designing the nanomagnets through proximity (tailoring the exchange interaction) and doping (e.g., with vanadium) to control the saturation magnetization, the nanomagnetic system can be made more robust to increased frequencies. The most important control parameter may be the Gilbert damping and this needs to be increased to stabilize the switching against random fluctuations. The damping can be engineered in magnetic media by adjustment of the fabrication conditions or the system topology. The samples can be prepared so 

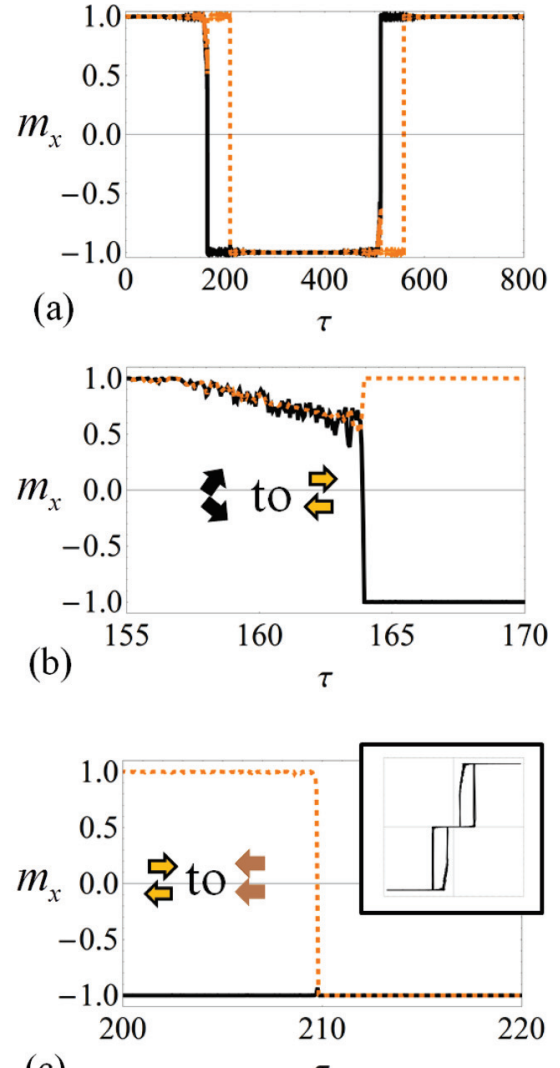

(c)

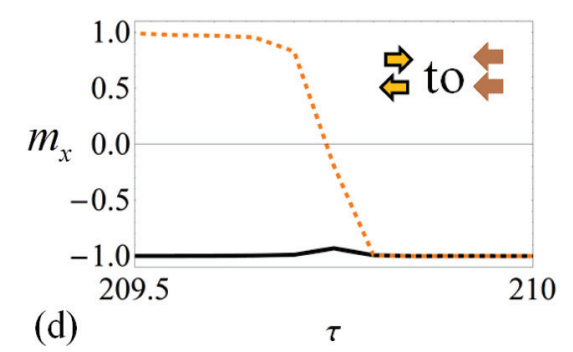

FIG. 12. (Color online) The switching times in the $A F 2$ state between scissored states and antiparallel states. The nanomagnets are taken to have dimensions $l_{x}=105 \mathrm{~nm}, l_{y}=13 \mathrm{~nm}$, and $l_{z}=2 \mathrm{~nm}$. The saturation magnetization is $M_{s}=5 \times 10^{5} \mathrm{~A} / \mathrm{m}$, the damping is $\alpha=0.01$, and $r=0.095$. The real time is given by $t=\left(\gamma M_{s}\right)^{-1} \tau$.

that very thin ferromagnetic resonance signatures exist and as such extrinsic sources of spin damping can be vastly reduced. ${ }^{42}$ Throughout this paper we have given examples with $\alpha=0.002$ to $\alpha=0.04$ to demonstrate that the predicted dynamical modes occur over a broad range of damping strengths. The elongated nanomagnets naturally have high levels of shape anisotropy. The magnitude of this anisotropy is determined by the structure and its strength can strongly influence the resonance frequencies of the system. ${ }^{43}$ The frequency linewidth is reduced as the aspect ratio of the nanomagnet increases. ${ }^{43}$ Nanomagnets with high structural quality exhibit very narrow ferromagnetic resonance (FMR) linewidths. ${ }^{44}$ The FMR linewidth can be related to the Gilbert damping parameter and the amount of inhomogeneity in the structure. ${ }^{44}$ The damping of the magnetization in the system is also directly related to the dissipation of energy into the thermal bath. The thickness of the nanomagnet is another factor to be taken into consideration when trying to tailor the damping. It was discovered by Liu and co-workers ${ }^{45}$ that the thickness can dictate the intrinsic and extrinsic dynamical properties of free layers of $\mathrm{CoFeB}$. The linewidth is commonly reported as $\Delta H \approx f \alpha / \gamma$ (e.g., Ref. 45). High-quality nanomagnets can be fabricated by a range of techniques, e.g., ultrahigh vacuum sputtering. ${ }^{44}$ The damping is affected by the surface roughness, volume defects, sample size, crystallinity, and magnetostatic interactions, to name a few influences. Thus, ensuring the quality of the samples is a means to lessen the extrinsic effects on the damping parameter (or indeed enhance them if one should so choose). ${ }^{46}$

For higher frequencies of the applied magnetic field the magnetization reversal process may be considerably lengthy. To resolve this issue the damping coefficient should be increased, leading to faster convergence of the system towards one of its stationary states. To optimize the performance of devices designed for memory elements, the duration of the applied magnetic field has to be defined by the switching time. $^{47}$ In Fig. 13(a) one can see that for a frequency of $1 \mathrm{GHz}$ the precession is reasonably smooth. Increasing the frequency of the applied field against the same sample with the same level of damping [Fig. 13(b)], the phase becomes purely $A F 2$ with a less smooth precessional characteristic. For the saturated magnetic states, where there are only parallel alignments of the magnetizations $\left(m_{x i}=1, m_{y i}=m_{z i}=0\right)$, the system is stable and evolves to one of two fixed point attractors. The excessive oscillations seen in Fig. 13(b) with thermal noise $r=0.122$ can be efficiently suppressed in the system with higher damping [see Fig. 13(c)], allowing one to bring the phase trajectory closer to the $x-y$ plane (and, therewith, indicating the validity for the usage of the quasistatic approximation for elongated nanomagnets) even for the case of thermal noise parameter $r=0.387$.

We wish to touch upon the evidence that can be seen throughout this work that phase locking can occur between the magnetizations in the nanomagnets, even with elevated thermal fluctuations. Most prominently this can be seen in the close examination of the magnetization evolution with time in Fig. 11 for the $A F 1$ phase. When the system resides firmly in a parallel state the magnetizations of the nanomagnets can be in synchronization. However, as the system evolves towards a scissored state, the synchronization is lost in the approach to the critical point of stability, where the system changes state. It would seem, that in order to synchronize the two nanomagnets, very precise and accurate control of the applied magnetic field has to be carried out.

It is considerably difficult to achieve full synchronization of magnetization dynamics, even in a system of two interacting macrospins, but under certain conditions such synchronized states can be found. For device applications under the finite temperatures it would be preferable to construct a robust system with prevailingly synchronized magnetization dynamics, which can be in part achieved by designing a proper energy landscape by varying the aspect ratio of the particles or the distance between them. In such an optimized system the contribution from the thermal noise can be made negligible, allowing one to achieve higher stability of magnetization dynamics. Our results offer valuable insights for such optimization; indeed, 
(a)
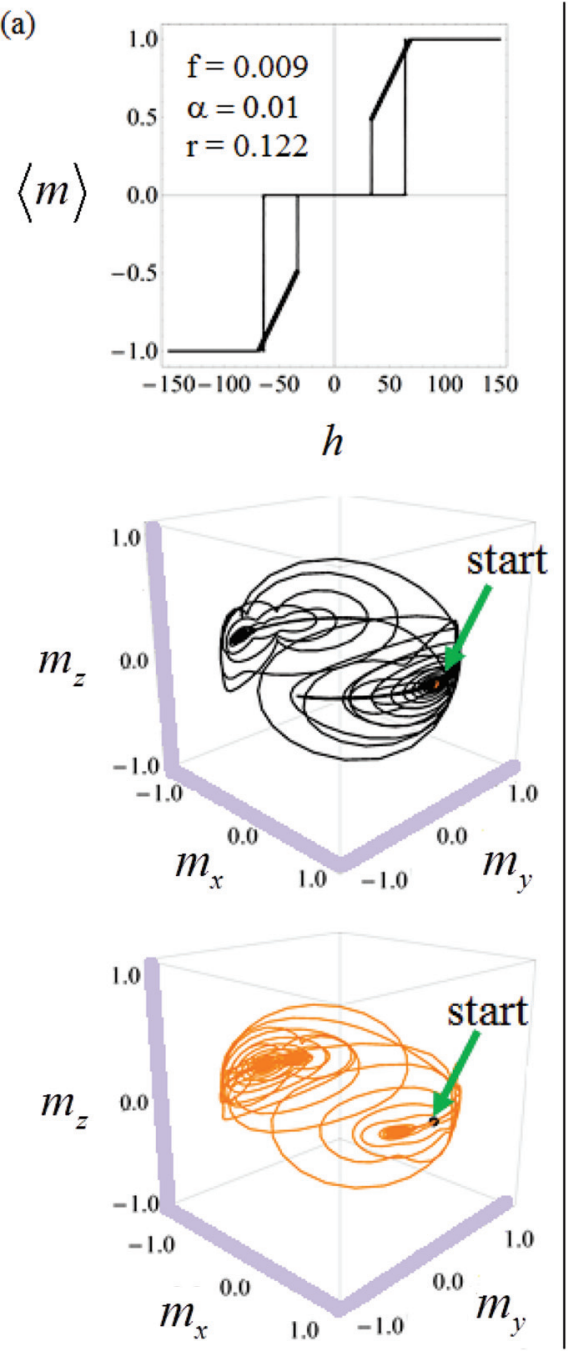

(b)
$\langle m\rangle$
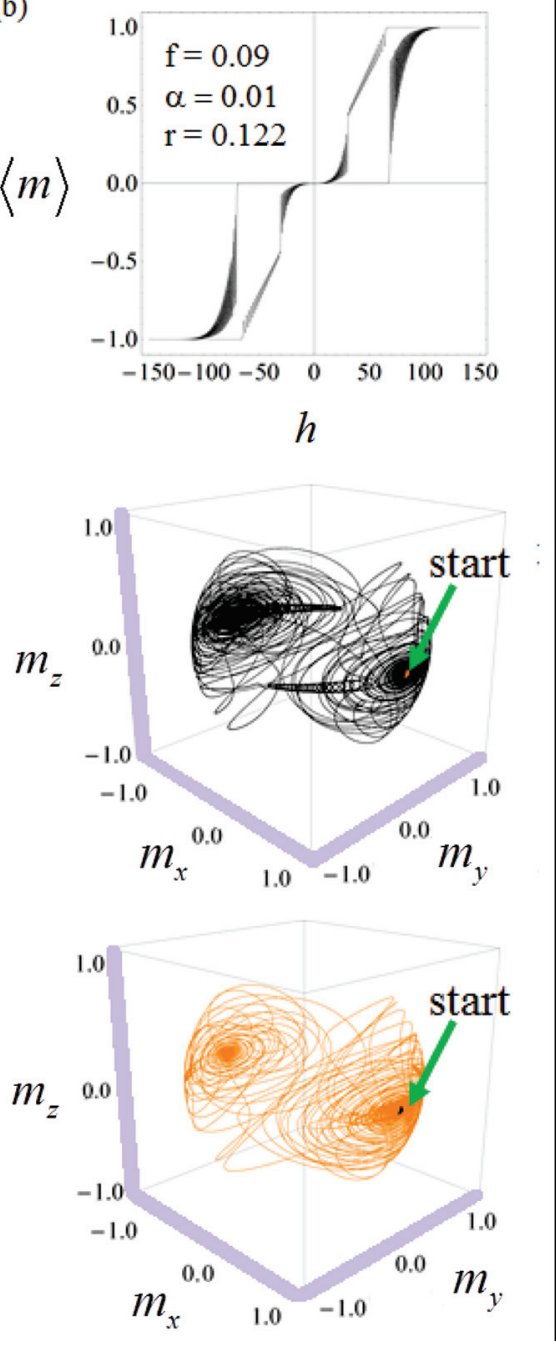

(c)
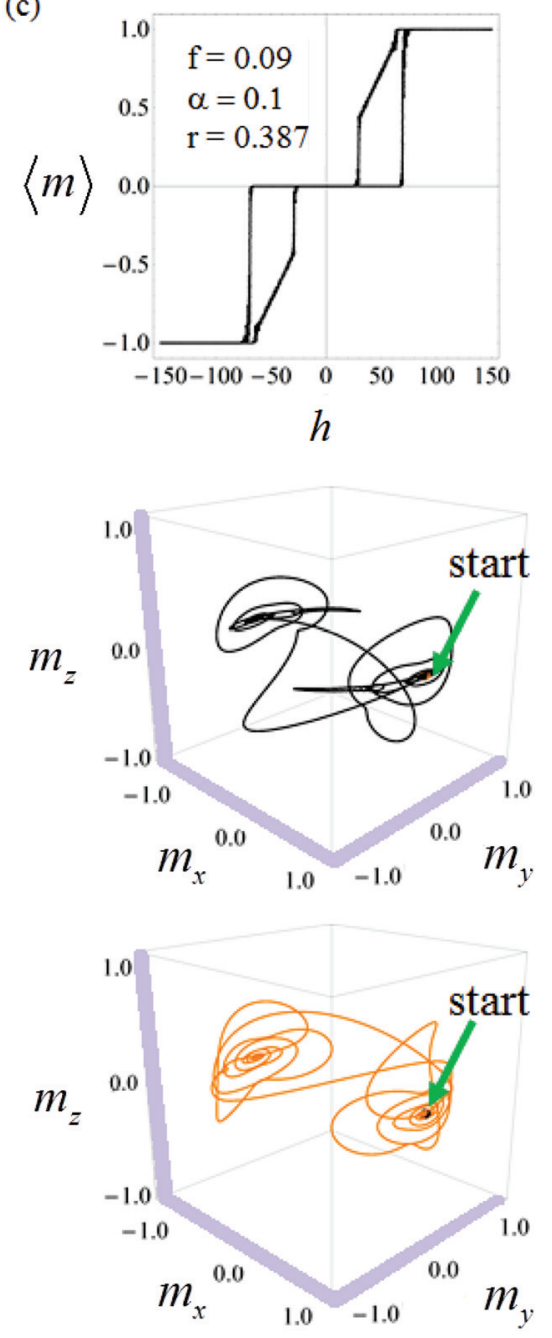

FIG. 13. (Color online) The magnetization precession of the nanomagnets in applied magnetic fields with frequencies of (a) $1 \mathrm{GHz}$ and [(b),(c)] $10 \mathrm{GHz}$. The nanomagnets are taken to have dimensions $l_{x}=75 \mathrm{~nm}, l_{y}=10 \mathrm{~nm}$, and $l_{z}=2 \mathrm{~nm}$. The saturation magnetization is $M_{s}=5 \times 10^{5} \mathrm{~A} / \mathrm{m}$ and the Gilbert damping is (a) $\alpha=0.01$, (b) $\alpha=0.01$, and (c) $\alpha=0.1$. In (a)-(c) the dimensionless units are $J=-50$, $a=-100 / 3$, and $b=-115$. The top plots in (a)-(c) are the average $x$-component magnetization as a function of the applied field strength $h$. Below each $\langle m\rangle$ versus $h$ plot is the precession of the magnetization of each individual nanomagnet. In (a) and (b), $r=0.122$ and in (c) $r=0.387$.

using the phase diagrams presented herein, one can focus on "problematic" points associated with considerable thermal fluctuations with the aim to reduce them by optimizing the shape of the nanomagnets and their mutual configuration or a distance between them. In such a way, some important results can be achieved, for example, in the development of wireless power transfer and/or communication systems using sensors and detectors which are operating with the usage of microwave oscillators composed of nanomagnets.

A full description of the synchronization of magnetic oscillators is outside the scope of this paper. But, indeed, a number of authors have reported on the difficulty of synchronizing nanomagnets and come up with novel solutions (e.g., nanomagnets driven by spin-polarized currents ${ }^{19,48,49}$ ). In our system, the magnetizations in both nanomagnets are free to rotate, not like in the spin-torque oscillators that have one ferromagnetic layer fixed. Systems with circular anisotropy seem most prone to exhibit periodic doubling towards chaos, ${ }^{50}$ but elongated samples are more robust on account of their high shape anisotropy. It is also noteworthy, that if the amplitude of the drive force is insufficient for a complete hysteresis cycle between $\langle m\rangle= \pm 1$, then the system finds new metastable states and chaos can ensue. For the interested reader, discussions on synchronization and chaos can be found in the work of Zhou, Persson, Åkerman and their co-workers. ${ }^{51-53}$ Also of significant interest, in the context of our work, is that it has been shown by Mitsumata and Tomita ${ }^{54}$ that the synchronization of a vibrating dipole field to the magnetization precession could be the origin of the variation in an effective damping parameter in a lattice of nanomagnets. The lattices that they considered contained "artificial atoms" that are made up of nanomagnets. In our work we have shown that the interaction of the nanomagnets is complicated even for two such components of a heterostructure. Our phase diagrams 
give valuable insights into the design of larger superlattices through identification of the design methodology to guard against disorder.

\section{DISCUSSION AND COMPARISON TO EXPERIMENTAL WORKS}

In many papers and reviews the $P$ and $A P$ phases appear. For example, in Ref. 55 the investigation of arrays of $\mathrm{FeSiB}$ microwires reveals a hysteresis profile similar in character to that of Fig. 8(c) that originates from the magnetization orientations in the two wires. In experimental analyses the Barkhausen jumps (which occur when the system experiences a transition between two metastable states) are measured as changes in the average magnetization due to the difficulties of characterizing the properties of an individual nanomagnet in a multimagnet array. Whenever the nanomagnets are sufficiently small or elongated, a series of magnetization plateaus and Barkhausen jumps are witnessed. ${ }^{56}$ In experiments where Barkhausen jumps are investigated, the metastable states of the system have a relative stability and to make the transition to another metastable state, the system has to surmount an energy barrier. ${ }^{57}$ The Zeeman energy dictates the transition between metastable states as the magnetic field is increased or decreased, but thermal fluctuations and the level of damping also hold sway (see Fig. 14). A random interaction field, as simulated by Eq. (16), has particular influence over a system near critical points of stability, i.e., near magnetic field strengths where Barkhausen jumps are emerging. Intriguingly, recent work ${ }^{58}$ has described the situation whereby switching occurs in the absence of an applied magnetic field and occurs due to the rapid heating of the thermal bath with a laser. Femtosecond laser pulses were fired into the thermal bath (of original temperature $300 \mathrm{~K}$ ) and the rapid transfer of thermal energy caused the switching of the magnetization in a few picoseconds. The authors of this work showed that the impetus towards magnetization reversal was unhindered even in a strong magnetic field. Thus, the phase diagrams of Figs. 1 and 2 can help assist in identifying at what magnetic field strengths an extra perturbation can be used to redefine the magnetic switching characteristics (an example of which is in Figs. 6-9). Magnetic devices must be designed with size, damping, and random fluctuations in mind and these phase diagrams provide a precursor to doing so. In particular, magnetic tunnel junctions are of considerable interest due to the possibility of functionalization as magnetic sensors and MRAM. This provides the motivation for most of the work appearing in the literature. The spin-flop mode of the $A F 1$ phase is clearly seen in the work of Fukumoto et $_{\text {al }}{ }^{26}$ where the samples were made by magnetron sputtering. The switching characteristics for antiferromagnetically coupled magnetic layers are difficult to obtain experimentally, but nevertheless there is a growing body of evidence of the viability of Worledge-like magnetic phases for toggle magnetic random access memories using elliptically shaped nanomagnets. ${ }^{59,60}$ Indeed, Kim and co-workers ${ }^{61}$ have investigated experimentally the effects of the thicknesses of the nanomagnets and spacer layers and they show the $A F 1$ phase for CoFeB SAF films of different heights. It should be noted that if the nanomagnets have different thicknesses, then the $A F 1$ hysteresis profile changes and appears similar in shape to the three nanomagnet hysteresis for magnetic elements of equal thicknesses (see, for example, Ref. 25). The change in thickness ratios is discussed in detail in Ref. 62. Our work highlights the importance of fine-tuning the system composed of two nanomagnets in order to design a robust device with a repetitive hysteresis curve under the action of thermal noise. The phase diagrams presented in the paper are useful for defining the parameter ranges required to obtain certain types and characteristics of magnetization reversal processes.

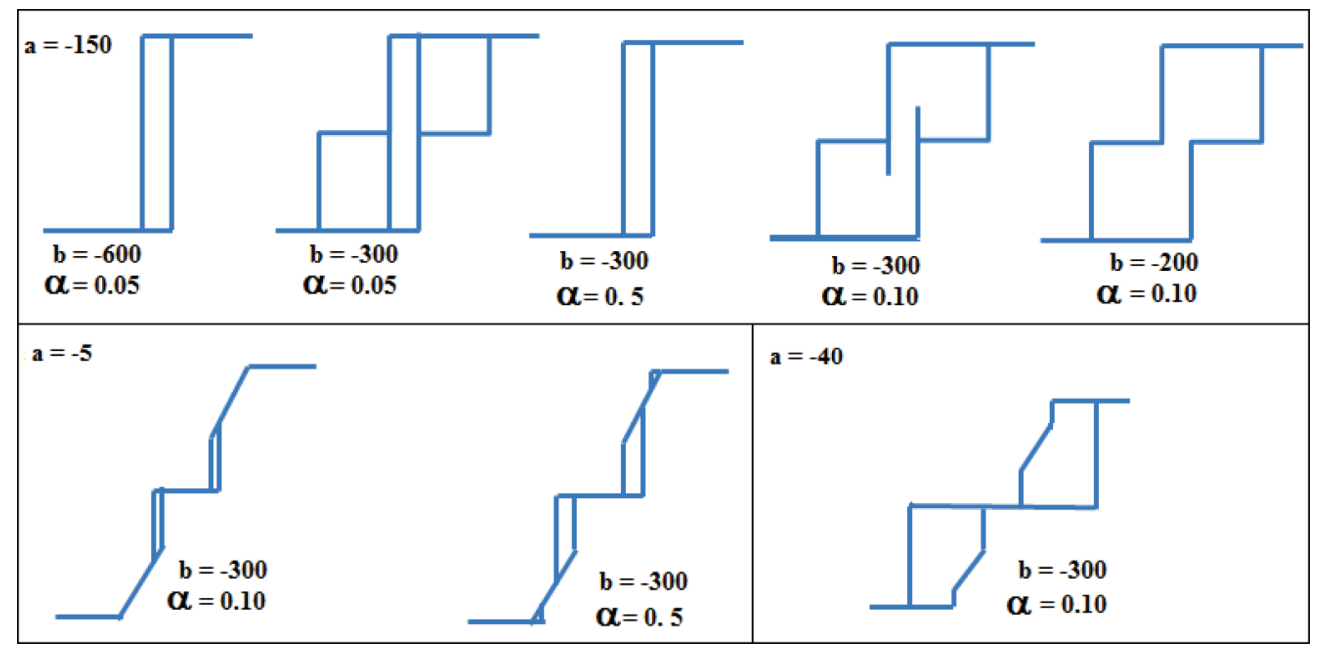

FIG. 14. (Color online) In this example, at $J=-40$ and $r=0.02$, the average magnetization as a function of the applied field changes with fluctuations of the Gilbert damping and the height of the energy barrier associated with anisotropy parameter $b$. The most variation occurs in the $A P$-type hysteresis curve, as shown in the top box for $a=-150$. The $A F$ phase hysteresis curves are more robust and can endure larger system changes. Changes in the damping strength can open up small hysteresis curves around the saturation field in the $A F 1$ phase and widen the existing hysteresis loops (shown for $a=-5$ ), but other than that the general characteristics are maintained. The $A F 2$ phase maintains its hysteresis shape with small movements of the maximum field strength associated with the antiparallel state, but is otherwise nonperturbed. 


\section{SUMMARY AND CONCLUSIONS}

In summary, we have studied the magnetization reversal of two interacting nanomagnets. In all examples we considered very elongated magnetic particles where their magnetic moments were oriented along the elongation axes. The two magnetic layers of doped $\mathrm{CoFeB}$ were coupled through a spacer layer that is composed of a material such as ruthenium. In the absence of an applied magnetic field the ground state for the two collinear magnetic particles corresponds to antiparallel ordering of magnetic moments arising due to their antiferromagnetic interaction. At large distances between particles this is dominated by dipole-dipole interaction. We have described a complex energy landscape, which consists of minima, maxima, and saddle points. The minima of this energy landscape correspond to different magnetic states. In the presence of an applied magnetic field there will arise other stable configurations of magnetic moments, such as parallel or scissored states.

The other parts of the energy landscape, such as the saddle points, are also very important since they determine the optimal paths of transitions between the magnetic states. Each optimal path of the transition between magnetic states is associated with the specific shape of the magnetic hysteresis loop. Here we presented a classification of possible hysteresis loops arising in the system of two interacting nanomagnets. These results have been first obtained with the use of a quasistatic approximation, where the energy landscape is well defined. Then we have studied the dynamical evolution of the nanoparticles magnetizations when the magnetic field was varying in the microwave $(\mathrm{GHz})$ range and compared the results obtained with the use of the quasistatic approximation. Specifically we have addressed the magnetization reversal in the nanosystem. We have estimated the characteristic time of the magnetization reversal of the system, when it is subjected to the microwave field, and found this to be of the order of $10^{-9} \mathrm{~s}$.

However, we have created phase diagrams to identify the magnetic properties of nanomagnetic systems based upon the shape of their magnetization curves as a function of the applied field strength. Within these magnetization curves there are hysteresis loops of varying sizes, the origins of which have been examined throughout. This work enables one to tailor the size of the hysteresis loop. The coupling strength, in a subtle balance with that of the anisotropy, can be used to expand or contract the width of a hysteresis loop. For example, the hysteresis loop can occur around a path between an antiparallel and a scissored magnetization state (the $A F 1$ phase) when the anisotropy is less than two-thirds of the coupling strength. Below this critical level reducing the anisotropy reduces the size of this hysteresis path (see Fig. 1). One may be interested in designing a nanomagnetic system that is controllable by a small applied magnetic field, where reaching the larger saturation field is undesirable, e.g., in the mechanical stimulation of the cellular membrane of a cancer cell. ${ }^{25}$ We identified that one can use a static magnetic field to "hold" the state of the nanomagnets magnetization before applying an alternating magnetic field with a much reduced amplitude to ensure that the system only operates around the favored hysteresis path. The static field is made half way between two critical points of stability and the maximum amplitude of the oscillating field need only be slightly greater than half of the difference between these points. We identified that these types of small hysteresis cycles can also be used for computational or memory operations with the different magnetization states used for binary logic (with consequently smaller switching times). We also found that the coupling strength of the nanoparticles is a very important parameter that influences the value of the switching time for a magnetization reversal and can also change the phase. For example, the reversal time to complete a full cycle of magnetization reversal and back increases in the $A P$ phase when the magnitude of the coupling strength of the particles increases, since in this case the barrier height separating the minima associated with magnetic states increases.

Throughout we have stressed the reliability of the quasistatic approximation to encapsulate the magnetization characteristics of single-domain interacting ferromagnetic systems within the range of damping found for SAF structures in experimental works (e.g., Refs. 14 and 15). We used this approximation, which is valid to give the first design step, before next performing a fully dynamical study. The occurrence of thermal fluctuations or a variation in the temperature, above zero kelvin, can change the characteristics of the energy landscape and develop other metastable states. At high frequencies the reliable and repeatable switching between parallel states can be jeopardized by the onset of chaotic transience. This can be countered by an increase in the damping. It is a fine balance that has to be found between the various competing energies and processes belonging to the system and also applied to the system. Thus, we found that in order to circumvent the difficulties of designing nanomagnetic devices it is perhaps prudent to first take the simplistic results from the quasistatic analysis and then knowing the basic character to undergo a dynamical simulation including damping and random fluctuations. It may then take several iterations of refinement to optimize the design to reach the shortest magnetization reversal times or regular magnetic oscillations. More clarity on what dictates the Gilbert damping parameters for a system consisting of many nanomagnets is required. However, it is hoped that the work presented here will provide insights on how dynamics and magnetization reversals occur in physical systems consisting of nanomagnetic elements and what parameters influence such processes.

We have determined the transitions between different states of magnetization using the forms of the hysteresis curves as the identifiers. We presented the phase diagrams, which have been developed using quasistatic techniques and the analytical solutions. The latter have provided analytical expressions for the critical lines of stability that mark the changes of magnetization states. In the present paper we have shown that by altering the strength of the magnetic coupling between the nanomagnets (for example, by changing a distance between them) we can bring the onset of different magnetic phases. Thus, the choice of the appropriate coupling or the distance between the particles can add or remove scissored states from the system, especially near the identified phase boundaries.

We have also shown that phases containing scissored states can be made to be very robust against thermal fluctuations, more so than those without them. This may happen when the 
damping increases or the particles become more elongated, for example. For phases containing no scissored states, i.e., the $A P$ phases, we have demonstrated the precarious nature of their hysteresis profile and a possible path to making them more stable. Again this may happen when the damping will be increased above some threshold value. In doing so, one could create nanomagnetic systems with very low coercive fields.

Thermal fluctuations alone are capable of switching the magnetization in a nanomagnetic system. ${ }^{58}$ Indeed, here we have shown the preservation of stable hysteresis cycles in the presence of varying levels of thermal noise. We have also demonstrated that the nanomagnets can be phase locked and brought into synchronization. This phenomenon may be useful in a construction of very powerful sources and/or highly sensitive antennas of microwave or even terahertz radiation. The high power or high sensitivity in such devices is reached when an array of such magnetic nanoparticles is synchronized. Designing functional structures that are composed of nanomagnets (largely through coupling and anisotropy parameters) has been seen in our description to be highly dependent upon the separation distances between the magnets, the actual geometries of the magnetic particles, their size, composition, and their orientation. These are also the principles for creating the structures known as metamaterials that control electromagnetic fields in ways unachievable by conventional materials. Thus, we predicate that the understanding developed here to optimize nanomagnetic structures in order to get the fastest magnetization reversal is also highly relevant to the design of future magnetic metamaterials.

\section{ACKNOWLEDGMENTS}

D.M.F. thanks the EPSRC for funding under KTA grant "Developing prototypes and a commercial strategy for nanoblade technology." E.K. thanks the TAMOP-4.2.1.B$10 / 2 / K O N V-2010-0001$ project in the framework of the New Hungarian Development Plan. We would also like to extend our gratitude to R. Giles for valuable assistance with the $\mathrm{C}$ programming language.
${ }^{1}$ T. X. Nan, Z. Y. Zhou, J. Lou, M. Liu, X. Yang, Y. Gao, S. Rand, and N. X. Sun, Appl. Phys. Lett. 100, 132409 (2012).

${ }^{2}$ D.-H. Kim, E. A. Rozhkova, I. V. Ulasov, S. D. Bader, T. Rajh, M. S. Lesniak, and V. Novosad, Nat. Mater. 9, 165 (2010).

${ }^{3}$ M. A. Ruderman and C. Kittel, Phys. Rev. 96, 99 (1954).

${ }^{4}$ T. Kasuya, Prog. Theor. Phys. 16, 45 (1956).

${ }^{5}$ K. Yosida, Phys. Rev. 106, 893 (1957).

${ }^{6}$ V. Tripathi, K. Dhochak, B. A. Aronzon, B. Raquet, V. V. Tugushev, and K. I. Kugel, Phys. Rev. B 85, 214401 (2012).

${ }^{7}$ D. C. Worldege, Appl. Phys. Lett. 84, 2847 (2004).

${ }^{8}$ H. Kubota, A. Fukushima, K. Yakushiji, S. Yakata, S. Yuasa, K. Ando, M. Ogane, Y. Ando, and T. Miyazaki, J. Appl. Phys 105, 07D117 (2009).

${ }^{9}$ K. Roy, S. Bandyopadhyay, J. Atulasimha, K. Munira, and A. W. Ghosh, arXiv:1107.0387v2.

${ }^{10}$ W. Hu, R. J. Wilson, A. Koh, A. Fu, A. Z. Faranesh, C. M. Earhart, S. J. Osterfeld, S.-J. Han, L. Xu, S. Guccione, R. Sinclair, and S. X. Wang, Adv. Mater. 20, 1479 (2008).

${ }^{11}$ Y. C. Kong, S. H. Lim, and K-J. Lee, J. Korean Phys. Soc. 54, 1630 (2009).

${ }^{12}$ W. Hu, R. J. Wilson, C. M. Earhart, A. L. Koh, R. Sinclair, and S. X. Wang, J. Appl. Phys. 105, 07B508 (2009).

${ }^{13}$ L. Zhou, J. Wiebe, S. Lounis, E. Vedmedenko, F. Meier, S. Blügel, P. H. Dederichs, and R. Wiesendanger, Nat. Phys. 6, 187 (2010).

${ }^{14}$ J. C. A. Huang, C. Y. Hsu, S. F. Chen, C. P. Liu, Y. F. Liao, M. Z. Lin, and C. H. Lee, J. Appl. Phys. 101, 123923 (2007).

${ }^{15}$ C.-W. Cheng, C. H. Shiue, T.-I. Cheng, and G. Chern, J. Appl. Phys. 112, 033917 (2012).

${ }^{16}$ R. Lai and A. J. Sievers, Phys. Rev. Lett. 81, 1937 (1998).

${ }^{17}$ K. Oguz, M. Ozdemir, O. Dur, and J. M. D. Coey, J. Appl. Phys. 111, 113904 (2012).

${ }^{18}$ M. Raoof, S. J. Corr, W. D. Kaluarachchi, K. L. Massey, K. Briggs, C. Zhu, M. A. Cheney, L. J. Wilson, and S. A. Curley,
Nanomedicine: Nanotechnology, Biology and Medicine 8, 1096 (2012).

${ }^{19}$ J. Xiao, A. Zangwill, and M. D. Stiles, Phys. Rev. B 72, 014446 (2005).

${ }^{20}$ F. M. Spedalieri, A. J. Jacob, D. E. Nikonov, and V. P. Roychowdhury, IEEE Trans. Nanotechnol. 10, 537 (2011).

${ }^{21}$ V. Tsiantos, W. Scholtz, D. Suess, T. Schrefl, and J. Fidler, J. Magn. Magn. Mater. 242-245, 999 (2002).

${ }^{22}$ K. Roy, S. Bandyopadhyay, and J. Atulasimha, J. Appl. Phys. 112, 023914 (2012).

${ }^{23}$ W. H. Press, S. A. Teukolsky, W. T. Vetterling, and B. P. Flannery, Numerical Recipes in C: The Art of Scientific Computing (Cambridge University Press, New York, 1992).

${ }^{24}$ W. F. Brown, Phys. Rev. 130, 1677 (1963).

${ }^{25}$ D. M. Forrester, K. E. Kürten, and F. V. Kusmartsev, Appl. Phys. Lett. 98, 163113 (2011).

${ }^{26}$ Y. Fukumoto, T. Suzuki, and S. Tahara, Appl. Phys. Lett. 89, 061909 (2006).

${ }^{27}$ N. Wiese, T. Dimopoulos, M. Rührig, J. Wecker, G. Reiss, J. Sort, and J. Nogues, Europhys. Lett. 78, 67002 (2007).

${ }^{28}$ M. N. Baibich, J. M. Broto, A. Fert, F. Nguyen, Van Dau, F. Petroff, P. Etienne, G. Creuzet, A. Friederich, and J. Chazelas, Phys. Rev. Lett. 61, 2472 (1988).

${ }^{29}$ G. Binasch, P. Grünberg, F. Saurenbach, and W. Zinn, Phys. Rev. B 39, 4828 (1989).

${ }^{30}$ D. M. Forrester, K. E. Kürten, and F. V. Kusmartsev, Phys. Rev. B 76, 134404 (2007).

${ }^{31}$ Y. Takahashi, Phys. Rev. B 56, 8175 (1997).

${ }^{32}$ V. V. Zalipaev, D. M. Forrester, C. M. Linton, and F. V. Kusmartsev, Localised States of Fabry-Perot Type in Graphene Nano-Ribbons, New Progress on Graphene Research, edited by Professor Jian Ru Gong (InTech, 2013).

${ }^{33}$ V. Zhukova, A. Zhukov, J. M. Blanco, J. Gonzalez, and B. K. Ponomarev, J. Magn. Magn. Mater. 249, 131 (2002). 
${ }^{34}$ V. I. Zdravkov, J. Kehrle, G. Obermeier, S. Gsell, M. Schreck, C. Müller, H.-A. Krug von Nidda, J. Lindner, J. Moosburger-Will, E. Nold, R. Morari, V. V. Ryazanov, A. S. Sidorenko, S. Horn, R. Tidecks, and L. R. Tagirov, Phys. Rev. B 82, 054517 (2010).

${ }^{35}$ A. A. Awad, A. Lara, V. Metlushko, K. Y. Guslienko, and F. G. Aliev, Appl. Phys. Lett. 100, 262406 (2012).

${ }^{36}$ R. P. Cowburn, A. O. Adeyeye, and M. E. Welland, New J. Phys. 1, 16 (1999).

${ }^{37}$ A. Chizhik and V. Zhukova, Physics Research International 2012, 690793 (2012).

${ }^{38}$ P. S. Keatley, V. V. Kruglyak, P. Gangmei, and R. J. Hicken, Philos. Trans. R. Soc. London, Ser. A 369, 3115 (2011).

${ }^{39}$ T. Kampfrath, A. Sell, G. Klatt, A. Pashkin, S. Mährlein, T. Dekorsy, M. Wolf, M. Fiebig, A. Leitenstorfer, and R. Huber, Nat. Photonics 5, 31 (2011).

${ }^{40}$ M. G. Benedict, P. Földi, and F. M. Peeters, J. Phys.: Conf. Ser. 36, 12 (2006).

${ }^{41}$ C. Scheck, L. Cheng, I. Barsukov, Z. Frait, and W. E. Bailey, Phys. Rev. Lett. 98, 117601 (2007).

${ }^{42}$ D. L. Mills and S. M. Rezende, in Spin Dynamics in Confined Magnetic Structures II, edited by B. Hillebrands and K. Ounadjela, Topics Appl. Phys. Vol. 87 (Springer-Verlag, Berlin Heidelberg, 2003), pp. 27-59.

${ }^{43}$ B. K. Kuanr, R. Lopusnik, and M. Wenger, J. Appl. Phys. 103, 07C508 (2008).

${ }^{44}$ A. N. Anisimov, W. Platow, P. Poulopoulos, M. Farle, K. Baberschke, P. Isberg, P. Granberg, and R. Wäppling, IEEE Trans. Magn. 34, 873 (1998).

${ }^{45}$ X. Liu, W. Zhang, M. J. Carter, and G. Xiao, J. Appl. Phys. 110, 033910 (2011).

${ }^{46}$ C. Vittoria, S. D. Yoon, and A. Widom, Phys. Rev. B 81, 014412 (2010).

${ }^{47}$ T. V. Lyutyy, A. Yu. Polyakov, A. V. Rot-Serov, and C. Binns, J. Phys.: Condens. Matter 21, 396002 (2009).
${ }^{48}$ S. I. Kiselev, J. C. Sankey, I. N. Krivorotov, N. C. Emley, R. J. Schoelkopf, R. A. Buhrman, and D. C. Ralph, Nature (London) 425, 380 (2003).

${ }^{49}$ P. Tabor, V. Tiberkevich, A. Slavin, and S. Urazhdin, Phys. Rev. B 82, 020407(R) (2010).

${ }^{50}$ R. K. Smith, M. Grabowski, and R. E. Camley, J. Magn. Magn. Mater. 322, 2127 (2010).

${ }^{51}$ Y. Zhou, J. Persson, and J. Åkerman, J. Appl. Phys. 101, 09A510 (2007).

${ }^{52}$ J. Persson, Y. Zhou, and J. Åkerman, J. Appl. Phys. 101, 09A503 (2007).

${ }^{53}$ Y. Zhou, H. Zhang, Y. Liu, and J. Åkerman, J. Appl. Phys. 112, 063903 (2012)

${ }^{54}$ C. Mitsumata and S. Tomita, Phys. Rev. B 84, 174421 (2011).

${ }^{55}$ L. C. Sampaio, E. H. C. P. Sinnecker, G. R. C. Cernicchiaro, M. Knobel, M. Vazquez, and J. Velazquez, Phys. Rev. B 61, 8976 (2000).

${ }^{56}$ L. Callegaro, E. Puppin, and M. Zani, J. Phys. D: Appl. Phys. 36, 2036 (2003).

${ }^{57}$ E. Puppin and M. Zani, J. Phys.: Condens. Matter 16, 1 (2004).

${ }^{58}$ T. A. Ostler, J. Barker, R. F. L. Evans, R. W. Chantrell, U. Atxitia, O. Chubykalo-Fesenko, S. El Moussaoui, L. Le Guyader, E. Mengotti, L. J. Heyderman, F. Nolting, A. Tsukamoto, A. Itoh, D. Afanasiev, B. A. Ivanov, A. M. Kalashnikova, K. Vahaplar, J. Mentink, A. Kirilyuk, Th. Rasing, and A. V. Kimel, Nat. Commun. 3, 666 (2012).

${ }^{59}$ D. C. Worledge, Via AP switching, Patent No. US 7, 002, 194 B2 (2006).

${ }^{60}$ A. Konovalenko, E. Lindgren, S. S. Cherepov, V. Korenivski, and D. C. Worledge, Phys. Rev. B 80, 144425 (2009).

${ }^{61}$ H.-J. Kim, S. C. Oh, J. S. Bae, K. T. Nam, J. E. Lee, S. O. Park, H. S. Kim, N. I. Lee, U.-In. Chung, J. T. Moon, and H. K. Kang, IEEE Trans. Magn. 41, 2661 (2005).

${ }^{62}$ H. Fujiwara, S.-Y. Wang, and M. Sun, J. Appl. Phys. 97, 10P507 (2005). 